\title{
Isospin effects on two-nucleon correlation functions in heavy-ion collisions at intermediate energies
}

\author{
Lie-Wen Chen,* V. Greco, and C. M. Ko \\ Cyclotron Institute and Physics Department, Texas A\&M University, College Station, Texas 77843-3366, USA
}

Bao-An Li

Department of Chemistry and Physics, P.O. Box 419, Arkansas State University, State University, Arkansas 72467-0419, USA

(Received 7 March 2003; revised manuscript received 24 April 2003; published 22 July 2003)

\begin{abstract}
Using an isospin-dependent transport model, we study isospin effects on two-nucleon correlation functions in heavy-ion collisions induced by neutron-rich nuclei at intermediate energies. We find that these correlation functions are sensitive to the density dependence of nuclear symmetry energy, but not to the incompressibility of symmetric nuclear matter and the medium dependence of nucleon-nucleon cross sections. This sensitivity is mainly due to effects of nuclear symmetry energy on the emission times of neutrons and protons as well as their relative emission sequence. We also study the variations of the symmetry energy effects on nucleonnucleon correlations with respect to the impact parameter, incident energy, and mass number of heavy-ion collisions.
\end{abstract}

DOI: 10.1103/PhysRevC.68.014605

PACS number(s): 25.70.Pq, 21.30.Fe, 21.65.+f, 24.10.Lx

\section{INTRODUCTION}

Heavy-ion collisions with radioactive nuclear beams provide a unique opportunity to study the properties of nuclear matter at the extreme condition of large isospin asymmetry [1-4]. In these collisions, the dynamics is affected not only by the isospin-independent part of nuclear equation of state (EOS) but also by the isospin-dependent part, i.e., the nuclear symmetry energy $E_{\text {sym }}(\rho)$, and the isospindependent in-medium nucleon-nucleon $(N N)$ cross sections. Knowledge on the density dependence of nuclear symmetry energy is essential for understanding both the structure of radioactive nuclei [5-8] and many key issues in astrophysics [9-11]. Although the root-mean-squared radius of neutron distribution in a heavy nucleus and that of a neutron star differ by about 18 orders of magnitude, the properties of both systems are strongly affected by the same $E_{\text {sym }}(\rho)[7,12,13]$. Moreover, the nucleosynthesis in presupernova evolution of massive stars, mechanisms of supernova explosions, cooling rates of protoneutron stars and associated neutrino fluxes, and the kaon condensation as well as the hadron to quarkgluon plasma phase transitions in neutron stars all rely critically on the nuclear symmetry energy $[9,10,14]$. However, our knowledge on $E_{\text {sym }}(\rho)$ is still rather poor, despite extensive theoretical studies based on various many-body theories [11]. In fact, it is often regarded as the most uncertain property of a neutron-rich matter $[15,16]$. Fortunately, radioactive beams, particularly the very energetic ones to be available at the planned rare isotope accelerator (RIA) and the new accelerator facility at the German Heavy Ion Accelerator Center, provide a great opportunity to study the $E_{\text {sym }}(\rho)$. It is thus important to investigate theoretically what experimental observables can be used to extract information about $E_{\text {sym }}(\rho)$. In this regard, significant progress has been made recently. For instance, measurement of neutron skins of ra-

\footnotetext{
* On leave from Department of Physics, Shanghai Jiao Tong University, Shanghai 200030, China.
}

dioactive nuclei via their total reaction cross sections [5] or that in stable heavy nuclei, such as ${ }^{208} \mathrm{~Pb}$, via parity-violating electron scatterings [7], was recently proposed as a sensitive probe to $E_{\text {sym }}(\rho)$. In heavy-ion collisions induced by neutron-rich nuclei, the pre-equilibrium neutron/proton ratio [17], isospin fractionation [18-22], isoscaling in multifragmentation [23], proton differential elliptic flow [24], neutronproton differential transverse flow [25], as well as the $\pi^{-}$to $\pi^{+}$ratio [26] have been identified as promising probes.

Observables mentioned in the above involve only the momentum, charge, and mass distributions of individual particles in the final state of a reaction. On the other hand, two-particle correlation functions, through final-state interactions and quantum statistics effects, have been shown to be a sensitive probe to the temporal and spatial information about the reaction dynamics in heavy-ion collisions at incident energies ranging from intermediate [27-29] to relativistic heavy-ion collider energy [30]. In particular, the correlation function of two nonidentical particles from heavy-ion collisions has been found to depend on their relative space-time distributions at freeze-out, and thus provides a useful tool for measuring the emission sequence, time delay, and separation between the emission sources for different particles [31-36]. Indeed, time delays as short as $1 \mathrm{fm} / c$ and source radius differences of a few tenths of a femtometer have been resolved experimentally from correlation functions of nonidentical particles [37].

In the present work, we study systematically how $E_{\text {sym }}(\rho)$ affects the temporal and spatial structure of reaction dynamics in heavy-ion collisions induced by neutron-rich nuclei at intermediate energies. A brief report of the present work can be found in Ref. [38]. We show that average emission times of neutrons and protons as well as their relative emission sequence in heavy-ion collisions are sensitive to $E_{\mathrm{sym}}(\rho)$. A stiffer density dependence of nuclear symmetry energy leads to faster and nearly simultaneous emissions of high momentum neutrons and protons. Consequently, strengths of the correlation functions for nucleon pairs with high total momentum, especially neutron-proton pairs with low relative 
momentum, are stronger for a stiffer $E_{\text {sym }}(\rho)$. This novel property thus provides another possible tool for extracting useful information about $E_{\text {sym }}(\rho)$. In addition, we also study how two-nucleon correlation functions depend on the isospin-independent part of nuclear matter EOS, the inmedium $N N$ cross sections, as well as the impact parameter, incident energy, and masses of colliding nuclei.

The paper is organized as follows. In Sec. II, we discuss the density dependence of nuclear symmetry energy and the EOS of neutron-rich nuclear matter. The isospin-dependent Boltzmann-Uehling-Uhlenbeck (IBUU) transport model used in the present study is briefly described in Sec. III. In Sec. IV, we presented results from the IBUU model on the nucleon emission functions and their isospin dependence in heavy-ion collisions induced by neutron-rich nuclei at intermediate energies. These include the nucleon emission times, the size of nucleon emission source, and the momentum distributions of emitted nucleons. In Sec. V, we study nucleonnucleon correlation functions and their dependence on the nuclear symmetry energy. We also present results on the variations of the symmetry energy effects on nucleonnucleon correlations with respect to the nuclear isoscalar potential, nucleon-nucleon cross sections, as well as the impact parameter, incident energy, and masses of heavy-ion collisions. Finally, we conclude with a summary and outlook in Sec. VI.

\section{NUCLEAR SYMMETRY ENERGY}

Many theoretical studies (e.g., Refs. [39-44]) have shown that the EOS of asymmetric nuclear matter can be approximately expressed as

$$
E(\rho, \delta)=E(\rho, \delta=0)+E_{\mathrm{sym}}(\rho) \delta^{2},
$$

where $\rho=\rho_{n}+\rho_{p}$ is the baryon density, $\delta=\left(\rho_{n}-\rho_{p}\right) /\left(\rho_{p}\right.$ $\left.+\rho_{n}\right)$ is the isospin asymmetry, and $E(\rho, \delta=0)$ is the energy per particle in symmetric nuclear matter. The bulk symmetry energy is denoted by the so-called symmetry energy coefficient $E_{\text {sym }}(\rho) \equiv E(\rho, \delta=1)-E(\rho, \delta=0)$. Its value at normal nuclear matter density $\rho_{0}$ [i.e., $E_{\text {sym }}\left(\rho_{0}\right)$ ] is predicted to be about $34 \pm 4 \mathrm{MeV}$, and is comparable to the value extracted from atomic mass data [45].

The symmetry energy coefficient $E_{\text {sym }}(\rho)$ can be expanded around normal nuclear matter density as

$$
E_{\mathrm{sym}}(\rho)=E_{\mathrm{sym}}\left(\rho_{0}\right)+\frac{L}{3}\left(\frac{\rho-\rho_{0}}{\rho_{0}}\right)+\frac{K_{\mathrm{sym}}}{18}\left(\frac{\rho-\rho_{0}}{\rho_{0}}\right)^{2}
$$

where $L$ and $K_{\text {sym }}$ are the slope and curvature of symmetry energy coefficient at normal nuclear density, i.e.,

$$
\begin{gathered}
L=\left.3 \rho_{0} \frac{\partial E_{\mathrm{sym}}(\rho)}{\partial \rho}\right|_{\rho=\rho_{0}}, \\
K_{\mathrm{sym}}=\left.9 \rho_{0}^{2} \frac{\partial^{2} E_{\mathrm{sym}}(\rho)}{\partial^{2} \rho}\right|_{\rho=\rho_{0}} .
\end{gathered}
$$

The $L$ and $K_{\text {sym }}$ characterize the density dependence of nuclear symmetry energy around normal nuclear matter density, and thus provide important information about the properties of nuclear symmetry energy at both high and low densities. They are, however, not well determined either theoretically or experimentally. The theoretically predicted values for $K_{\text {sym }}$ vary from about $-700 \mathrm{MeV}$ to $+466 \mathrm{MeV}$ (e.g., Ref. [46]), while data from giant monopole resonances indicate that $K_{\text {sym }}$ is in the range from $-566 \pm 1350 \mathrm{MeV}$ to $34 \pm 159 \mathrm{MeV}$, depending on the mass region of nuclei and the number of parameters used in parametrizing the incompressibility of finite nuclei [47]. There are no experimental data for the value of $L$, and different theoretical models give its value from -50 to $200 \mathrm{MeV}$ [8].

Predictions for the density dependence of $E_{\text {sym }}(\rho)$ are thus divergent. In the present study, we adopt the parametrization used in Ref. [48] for studying the properties of neutron stars, i.e.,

$$
E_{\mathrm{sym}}(\rho)=E_{\mathrm{sym}}\left(\rho_{0}\right) u^{\gamma}
$$

where $u \equiv \rho / \rho_{0}$ is the reduced density and $E_{\text {sym }}\left(\rho_{0}\right)$ $=35 \mathrm{MeV}$ is the symmetry energy at normal nuclear matter density. The symmetry potential acting on a nucleon derived from the above nuclear symmetry energy [24] is

$$
\begin{aligned}
V_{\mathrm{sym}}(\rho, \delta)= & \pm 2\left[E_{\mathrm{sym}}\left(\rho_{0}\right) u^{\gamma}-12.7 u^{2 / 3}\right] \delta \\
& +\left[E_{\mathrm{sym}}\left(\rho_{0}\right)(\gamma-1) u^{\gamma}+4.2 u^{2 / 3}\right] \delta^{2},
\end{aligned}
$$

where "+" and "-" are for neutrons and protons, respectively.

For the isospin-independent part of the nuclear EOS, $E(\rho, \delta=0)$, we use a Skyrme-like parametrization, i.e.,

$$
E(\rho, \delta=0)=\frac{3}{5} E_{F}^{0} u^{2 / 3}+\frac{a}{2} u+\frac{b}{1+\sigma} u^{\sigma},
$$

where the first term is the kinetic part and $E_{F}^{0}$ is the Fermi energy of symmetric nuclear matter at normal nuclear matter density. The parameters $a=-358.1 \mathrm{MeV}, b=304.8 \mathrm{MeV}$, and $\sigma=7 / 6$ correspond to the so-called soft EOS with incompressibility $K_{0}=201 \mathrm{MeV}$, while $a=-123.6 \mathrm{MeV}, b$ $=70.4 \mathrm{MeV}$, and $\sigma=2$ give the so-called stiff EOS with incompressibility $K_{0}=380 \mathrm{MeV}$. Both EOS's have a saturation density $\rho_{0}=0.16 \mathrm{fm}^{-3}$ for symmetric nuclear matter. The isospin-independent potential obtained from this EOS is then

$$
V_{0}(\rho)=a u+b u^{\sigma} .
$$

The pressure of nuclear matter is given by

$$
P(\rho, \delta)=\rho^{2} \frac{\partial E(\rho, \delta)}{\partial \rho},
$$

which leads to a contribution from nuclear symmetry energy given by

$$
P_{\mathrm{sym}}(\rho, \delta)=\rho^{2} \delta^{2} \frac{\partial E_{\mathrm{sym}}(\rho)}{\partial \rho}=\rho_{0} E_{\mathrm{sym}}\left(\rho_{0}\right) \gamma \delta^{2} u^{\gamma+1},
$$


besides that due to isospin-independent energy given by

$$
P_{0}(\rho, \delta=0)=\frac{2}{5} E_{F}^{0} \rho_{0} u^{5 / 3}+\frac{1}{2} a \rho_{0} u^{2}+\frac{b \sigma}{1+\sigma} u^{\sigma+1} .
$$

At moderate and high temperatures $T(T>4 \mathrm{MeV})$, the pressure can also be decomposed into an isospin-dependent part $P_{\text {sym }}(\rho, \delta, T)$ and an isospin-independent part $P_{0}(\rho, \delta$ $=0, T)$, i.e.,

$$
\begin{aligned}
P_{\mathrm{sym}}(\rho, \delta, T)= & \frac{1}{2} \sum_{n=1}^{\infty} b_{n}\left(\frac{\lambda_{T}^{3} \rho}{4}\right)^{n}\left[(1+\delta)^{1+n}+(1-\delta)^{1+n}\right. \\
& -2]+\rho_{0} \delta^{2}\left[E_{\mathrm{sym}}\left(\rho_{0}\right) \gamma u^{\gamma+1}-8.5 u^{5 / 3}\right]
\end{aligned}
$$

and

$$
\begin{aligned}
P_{0}(\rho, \delta=0, T)= & T \rho\left[1+\sum_{n=1}^{\infty} b_{n}\left(\frac{\lambda_{T}^{3} \rho}{4}\right)^{n}\right]+\frac{1}{2} a \rho_{0} u^{2} \\
& +\frac{b \sigma}{1+\sigma} u^{\sigma+1},
\end{aligned}
$$

where $b_{n}^{\prime}$ s are the inversion coefficients given in Refs. $[18,3]$ and

$$
\lambda_{T}=\left(\frac{2 \pi \hbar^{2}}{m T}\right)^{1 / 2}
$$

is the thermal wavelength of nucleons with mass $m$. In Eq. (12), we have used the explicit $\delta$-dependent kinetic part of symmetry energy instead of the parabolic approximation to calculate the pressure at finite temperatures.

In the present work, we consider two cases of $\gamma=0.5$ (soft) and 2 (stiff) to explore the large range of $L$ and $K_{\text {sym }}$ discussed above. The $\gamma=0.5$ (2) gives $L=52.5$ (210.0) $\mathrm{MeV}$ and $K_{\text {sym }}=-78.8$ (630.0) MeV. In Figs. 1(a) and 1(b), we show the nuclear symmetry potentials for protons and neutrons in isospin asymmetric nuclear matter with $\delta=0.2$ and 0.4 , respectively. Except at densities below $0.5 \rho_{0}$ for the case of $\gamma=2$, the symmetry potential is attractive for protons and repulsive for neutrons, and increases with density and isospin asymmetry. Furthermore, the stiff symmetry potential becomes stronger than the soft one when the nuclear density exceeds certain value around normal nuclear density, which depends on the isospin asymmetry and is different for neutrons and protons.

Different symmetry energies also result in different thermodynamical properties for neutron-rich matter. The pressure due to symmetry energy, $P_{\text {sym }}$, in an asymmetric nuclear matter with isospin asymmetry $\delta=0.2$ or 0.4 and temperature $T=0$ or $10 \mathrm{MeV}$, is shown in Fig. 2 as a function of density for both soft $(\gamma=0.5)$ and stiff $(\gamma=2)$ symmetry energies. It is seen that the stiff symmetry energy gives a larger pressure in asymmetric nuclear matter than the soft one at the same density, and the difference increases with both density and isospin asymmetry. Since the symmetry en-

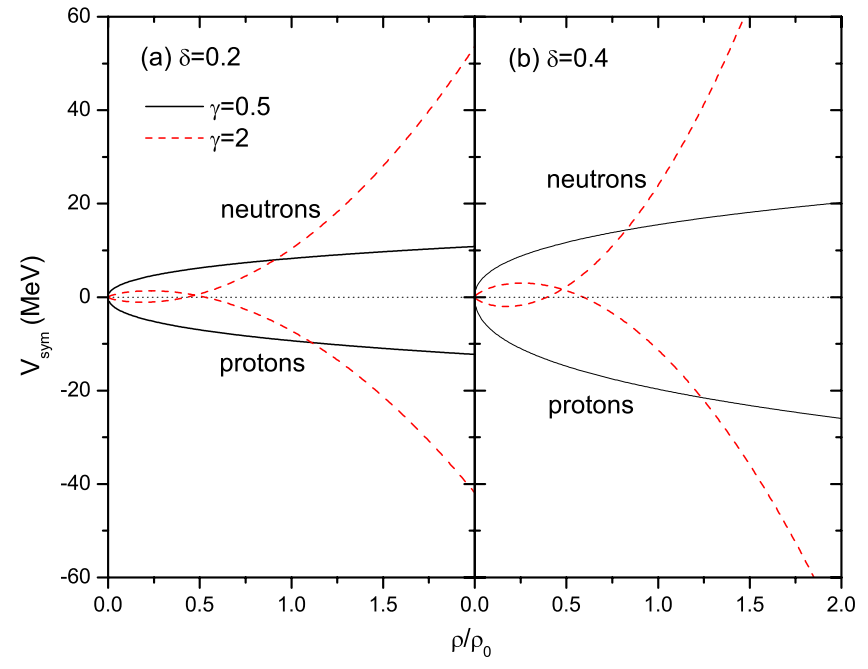

FIG. 1. (Color online) Neutron and proton symmetry potentials as functions of density in asymmetric nuclear matter with isospin asymmetry (a) $\delta=0.2$ and (b) $\delta=0.4$ for both stiff $(\gamma=2)$ and soft $(\gamma=0.5)$ symmetry energies, respectively.

ergy is insensitive to temperature, temperature dependence of the pressure from symmetry energy is rather weak, as in the more sophisticated finite-temperature Skyrme-Hartree-Fock calculations [49]. For comparison, the isospin-independent part of pressure, $P_{0}$, is shown in Fig. 3 as a function of density for (a) $T=0$ and (b) $10 \mathrm{MeV}$, and with $K_{0}=201$ (dashed curves) and 380 (solid curves) MeV. We see that the stiff EOS gives a stronger pressure at both higher densities and temperatures.

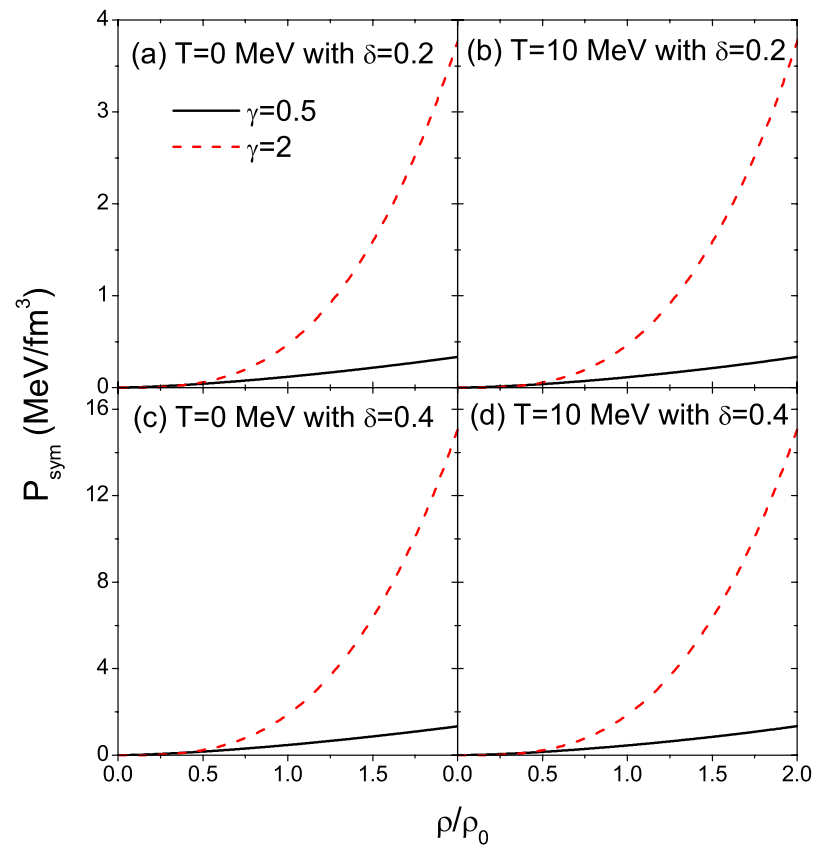

FIG. 2. (Color online) Pressure of asymmetric nuclear matter due to the symmetry energy, $P_{\text {sym }}$, as a function of density for different temperatures and isospin asymmetries: (a) $T=0 \mathrm{MeV}$ and $\delta=0.2$, (b) $T=10 \mathrm{MeV}$ and $\delta=0.2$, (c) $T=0 \mathrm{MeV}$ and $\delta=0.4$, and (d) $T=10 \mathrm{MeV}$ and $\delta=0.4$. Solid and dashed curves are for soft $(\gamma=2.0)$ and stiff $(\gamma=0.5)$ symmetry energies, respectively. 


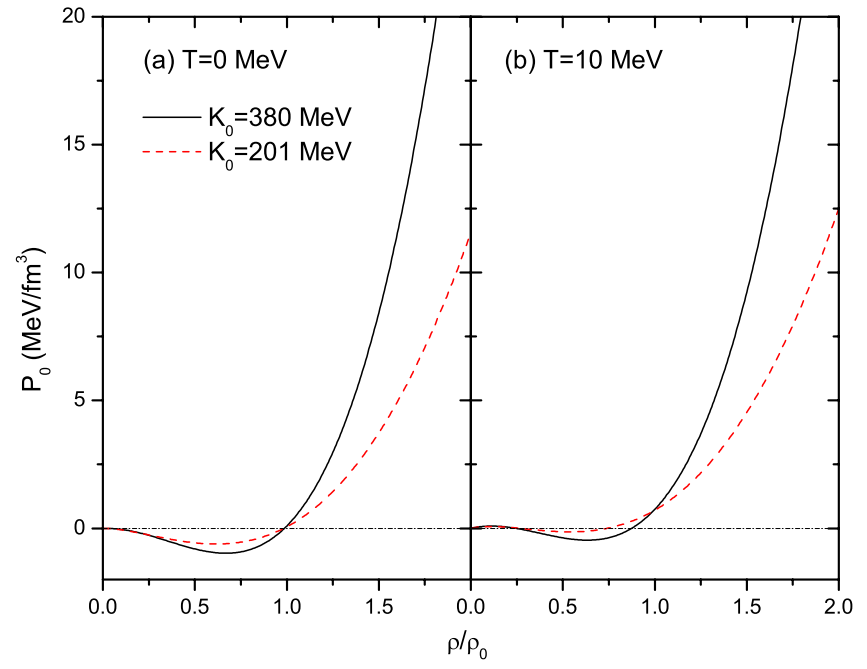

FIG. 3. (Color online) Isospin-independent part of pressure, $P_{0}$, as a function of density for (a) $T=0$ and (b) $10 \mathrm{MeV}$, respectively, with $K_{0}=380$ (solid curves) and 201 (dashed curves) MeV.

Although the total incompressibility of asymmetric nuclear matter is mainly determined by the stiffness of the isoscalar part of nuclear energy, the stiffness of symmetry energy has non-negligible effects on its EOS and thermodynamical properties. We thus expect that different symmetry energies would lead to different space-time evolutions in heavy-ion collisions induced by neutron-rich nuclei at intermediate energies, resulting in observable effects in the nucleon-nucleon correlation functions.

\section{ISOSPIN-DEPENDENT BUU TRANSPORT MODEL}

Our study is based on an IBUU transport model (e.g., Refs. [50,17,25,26,51]). In this model, initial positions of protons and neutrons are determined according to their density distributions from the relativistic mean-field theory [5254]. The neutron and proton initial momenta are then taken to have uniform distributions inside their respective Fermi spheres, with Fermi momenta determined by their local densities via the Thomas-Fermi approximation. For the isoscalar potential, we use as default the Skyrme potential with incompressibility $K_{0}=380 \mathrm{MeV}$. Although the transverse flow data from heavy-ion collisions are best described by a momentum-dependent soft potential with $K_{0}=210 \mathrm{MeV}$ $[55,56]$, they can also be approximately reproduced with a momentum-independent stiff potential with $K_{0}=380 \mathrm{MeV}$. Dependence of the reaction dynamics on isospins is included through the isospin-dependent total and differential nucleonnucleon cross sections and Pauli blockings, the symmetry potential $V_{\text {sym }}$, and the Coulomb potential $V_{\mathrm{c}}$ for protons. For nucleon-nucleon cross sections, we use as default the experimental values in free space, $\sigma_{\text {exp }}$. For a review of the IBUU model, we refer the reader to Ref. [3]. To study effects due to the medium dependence of nucleon-nucleon cross sections, we also use the parametrized in-medium nucleonnucleon cross sections $\sigma_{\text {in-medium }}$ from the Dirac-Brueckner approach based on the Bonn- $A$ potential [57]. In Figs. 4 (a)(c), we show the density dependence of total nucleon-

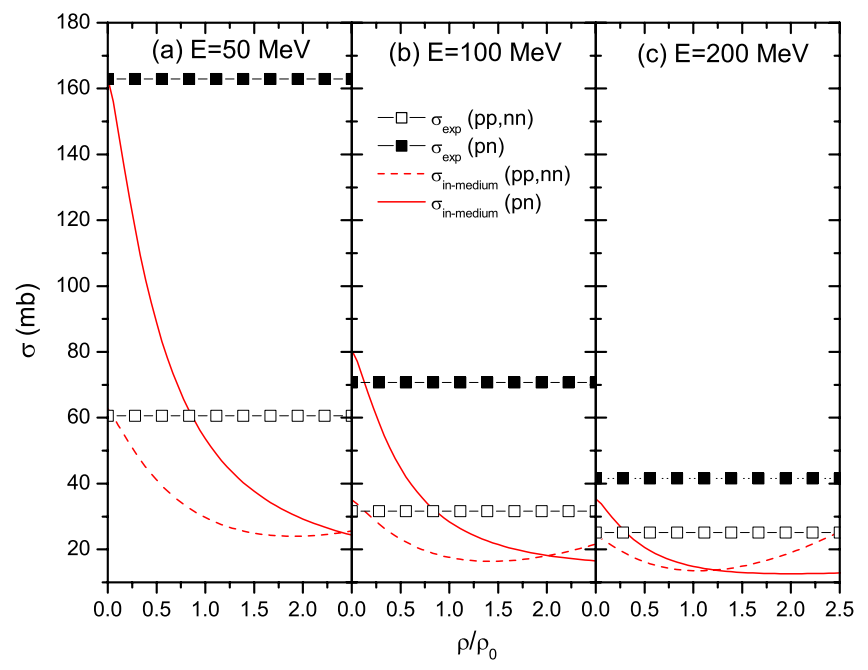

FIG. 4. (Color online) Density dependence of total nucleonnucleon cross sections at laboratory energies (a) $E=50$, (b) 100 and (c) $200 \mathrm{MeV}$, for the experimental free-space cross sections (squares) and the in-medium nucleon-nucleon cross sections from the Dirac-Brueckner approach based on the Bonn- $A$ potential (lines).

nucleon cross sections $\sigma_{\text {exp }}$ (squares) and $\sigma_{\text {in-medium }}$ (lines) for laboratory incident energies $E=50,100$, and $200 \mathrm{MeV}$, respectively. In experimental free-space cross sections, the neutron-proton cross section is about a factor of 3 larger than the neutron-neutron or proton-proton cross section. On the other hand, in-medium nucleon-nucleon cross sections from the Dirac-Brueckner approach based on the Bonn- $A$ potential have smaller magnitude and weaker isospin dependence than $\sigma_{\text {exp }}$, but strong density dependence.

To solve the IBUU model, we use the usual test particle method [50]. Results presented in the following are obtained with 10000 events using 100 test particles for a physical nucleon.

\section{NUCLEON EMISSION FUNCTIONS}

The nucleon emission function describes the distributions of time, position, and momentum at which nucleons are emitted. In the following, we first show the distribution of nucleon emission times and the dependence of the average emission time on the nucleon momentum. This is then followed by the distribution of nucleon emission positions and its dependence on the nucleon momentum. The momentum distribution of emitted nucleons is also given. All momenta are in the center-of-mass system unless stated otherwise.

\section{A. Nucleon emission times}

The emission times of different particles in heavy-ion collisions are relevant for understanding both the collision dynamics and the mechanism for particle production. In heavyion collisions at intermediate energies, the dynamics of nucleon emissions is mainly governed by the pressure of the excited nuclear matter produced during the initial stage of collisions $[24,58]$. Since the stiff symmetry energy gives a 


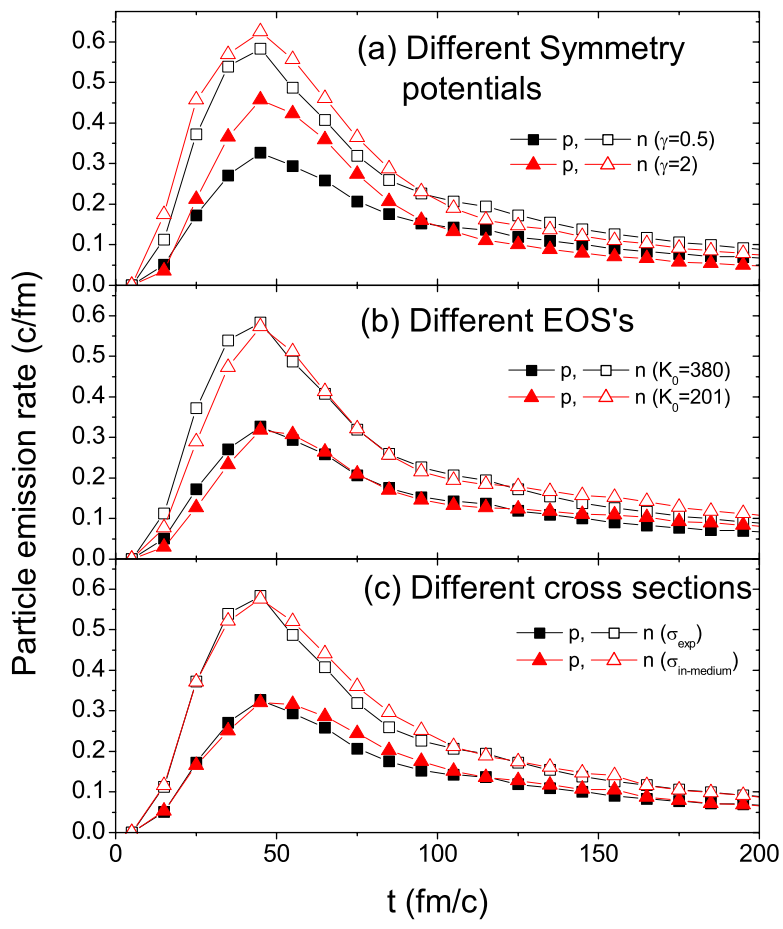

FIG. 5. (Color online) Emission rates of protons and neutrons as functions of time for different cases: (a) Soft or stiff symmetry energy with $K_{0}=380 \mathrm{MeV}$ and $\sigma_{\text {exp }}$, (b) different EOS's, i.e., $K_{0}$ $=380$ and $200 \mathrm{MeV}$ with $\gamma=0.5$, and (c) different $N N$ cross sections with $K_{0}=380 \mathrm{MeV}$ and $\gamma=0.5$.

larger pressure than that due to the soft symmetry energy as shown in Fig. 2, it leads to a faster emission of neutrons and protons. The relative emission sequence of neutrons and protons is, however, determined by the difference in their symmetry potentials. With the symmetry potential being generally repulsive for neutrons and attractive for protons, neutrons are expected to be emitted earlier than protons. The difference in neutron and proton emission times is particularly large for the soft symmetry potential as its magnitude at densities below the normal nuclear matter density, where most nucleons are emitted, is larger than that of stiff symmetry potential as seen in Fig. 1. Since the stiff symmetry potential changes sign when the nuclear density drops below $0.5 \rho_{0}$, the relative emission sequence of neutrons and protons in this case depends on the average density at which they are emitted. Also, the emission time for protons is affected by the repulsive Coulomb potential. Of course, details of these competing effects on nucleon emissions depend on both the reaction dynamics and momenta of nucleons. These details can only be studied by using transport models.

As an example, we study here central collisions of ${ }^{52} \mathrm{Ca}$ $+{ }^{48} \mathrm{Ca}$ at $E=80 \mathrm{MeV} /$ nucleon. This particular reaction system with isospin asymmetry $\delta=0.2$ can be studied at the RIA. In the present work, nucleons are considered as being emitted when their local densities are less than $\rho_{0} / 8$ and subsequent interactions do not cause their recapture into regions of higher density. Other emission criteria, such as taking the nucleon emission time as its last collision time in the IBUU model, do not change our conclusions. In Fig. 5, we show the emission rates of protons and neutrons as functions of time for different cases. It is clearly seen that there are two stages of nucleon emissions; an early fast emission and a subsequent slow emission. This is consistent with the long-lived nucleon emission source observed in previous BUU calculations [59]. In Fig. 5(a), effects due to different symmetry energies are shown, and we find that the stiff symmetry energy (triangles) enhances the emission of early high momentum protons and neutrons but suppresses late slow emission compared with results from the soft symmetry energy (squares). Difference between the emission rates of protons and neutrons is, however, larger for the soft symmetry energy. Both features are what we have expected from the above discussions about the effects of nuclear symmetry energy on particle emissions. Figure 5(b) shows results by using different incompressibilities of $K_{0}=380$ (squares) and 201 (triangles) $\mathrm{MeV}$ for the isoscalar potential. It is seen that the nucleon emission rate is not sensitive to the incompressibility $K_{0}$ of the symmetric nuclear matter EOS, except that the stiff EOS slightly enhances the nucleon emission rate at the early stage of reactions. In Fig. 5(c), we compare results from the experimental free space $N N$ cross section $\sigma_{\exp }$ (squares) and those from the in-medium $N N$ cross sections $\sigma_{\text {in-medium }}$ (triangles). Again, the nucleon emission rate is found to be insensitive to the $N N$ cross sections used for the present reaction system, except that the in-medium cross sections slightly enhance the nucleon emission rate at later stage of reactions $(50-100 \mathrm{fm} / c)$.

To see if nucleons are emitted from an equilibrium or nonequilibrium sources, we consider time evolution of the scaled quadrupole moment of nucleon momentum distribution $Q_{\mathrm{zz}} / A_{\text {res }}$ defined as [60]

$$
Q_{\mathrm{zz}} / A_{\mathrm{res}}(t)=1 / A_{\text {res }} \int \frac{d \mathbf{r} d \mathbf{p}}{(2 \pi)^{3}}\left(2 p_{z}^{2}-p_{x}^{2}-p_{y}^{2}\right) f(\mathbf{r}, \mathbf{p}, \mathbf{t}),
$$

where $A_{\text {res }}$ is the mass number of the residue composed of nucleons at local density larger than $\rho_{0} / 8$, and $f(\mathbf{r}, \mathbf{p}, \mathbf{t})$ is the nucleon phase-space distribution function given by the IBUU model. How far the emission source deviates from thermal equilibrium is then given by the value of $Q_{\mathrm{zz}}$, with $Q_{\mathrm{zz}}=0$ implying that thermal equilibrium is achieved. Shown in Fig. 6 are time evolutions of $Q_{z z} / A_{\text {res }}$ for different nuclear symmetry potentials, isoscalar potentials, and $N N$ cross sections. It is seen that the emission source nearly reaches thermal equilibrium after around $40 \mathrm{fm} / c$, except the case with inmedium $N N$ cross sections (dash-dotted curve). In the latter case, the thermal equilibration time is delayed to about $70 \mathrm{fm} / c$ as a result of smaller in-medium $N N$ cross sections than free $N N$ cross sections. Therefore, early emitted nucleons are mostly from pre-equilibrium stage of heavy-ion collisions, while late emitted ones are more like statistical emissions. Since positive $Q_{z z}$ indicates incomplete nuclear stopping or nuclear transparency while negative one implies transverse expansion or collectivity, all cases except the one with in-medium $N N$ cross sections thus show strong nuclear stopping but weak transverse expansion after $120 \mathrm{fm} / c$.

The weak dependence of the nucleon emission rate on the stiffness of isoscalar potential is due to reduced difference in 


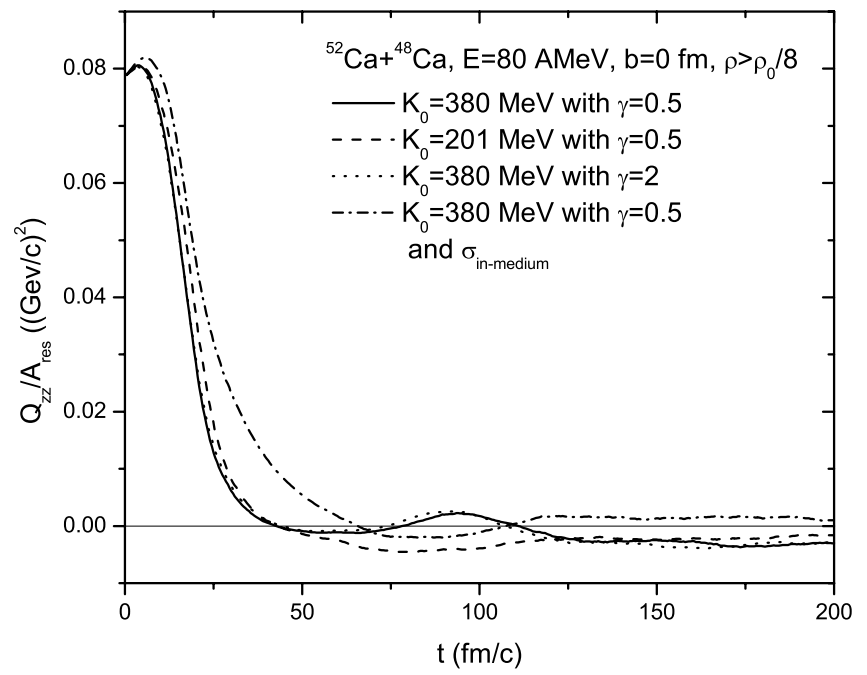

FIG. 6. Time evolutions of scaled quadrupole moment of nucleon momentum distribution $Q_{z z} / A_{\text {res }}$ by using $K_{0}=380$ and $210 \mathrm{MeV}$ with $\gamma=0.5, K_{0}=380$ with $\gamma=2$, and $K_{0}=380 \mathrm{MeV}$, with $\gamma=0.5$ and in-medium $N N$ cross sections.

the pressure of excited nuclear matter as a result of different maximum densities reached in collisions, with the stiff one giving a lower density than the soft one. This can be seen in Fig. 7 where the time evolution of average central density (calculated in a sphere located at the center of colliding nuclear matter with radius of $3 \mathrm{fm}$ ) is shown by using $K_{0}$ $=380$ (solid curve) or 201 (dashed curve) $\mathrm{MeV}$, but with same soft symmetry energy and free nucleon-nucleon cross sections. Maximum average central densities reached in the collisions are about $1.46 \rho_{0}$ and $1.66 \rho_{0}$, respectively, for $K_{0}$ $=380$ and $201 \mathrm{MeV}$, resulting in similar pressures of about 8 and $7 \mathrm{MeV} / \mathrm{fm}^{3}$, respectively. In addition, the soft EOS leads to a slower expansion and a longer higher density stage than the stiff EOS. On the other hand, the maximum average central density reached in the collisions is reduced only

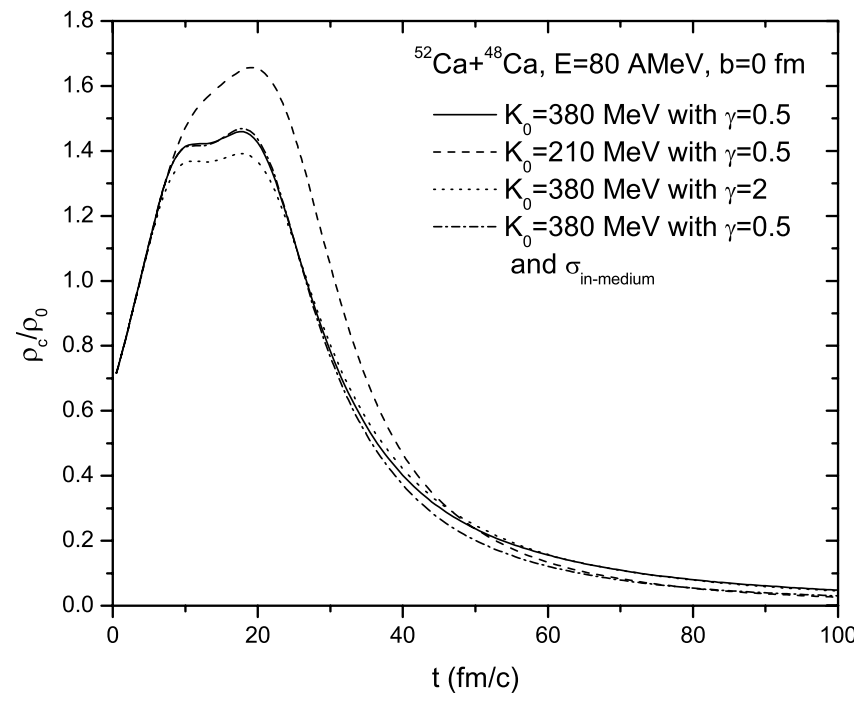

FIG. 7. Time evolution of average central density by using $K_{0}$ $=380$ and $210 \mathrm{MeV}$ with $\gamma=0.5, K_{0}=380$ with $\gamma=2$, and $K_{0}$ $=380 \mathrm{MeV}$ with $\gamma=0.5$ and in-medium $N N$ cross sections.

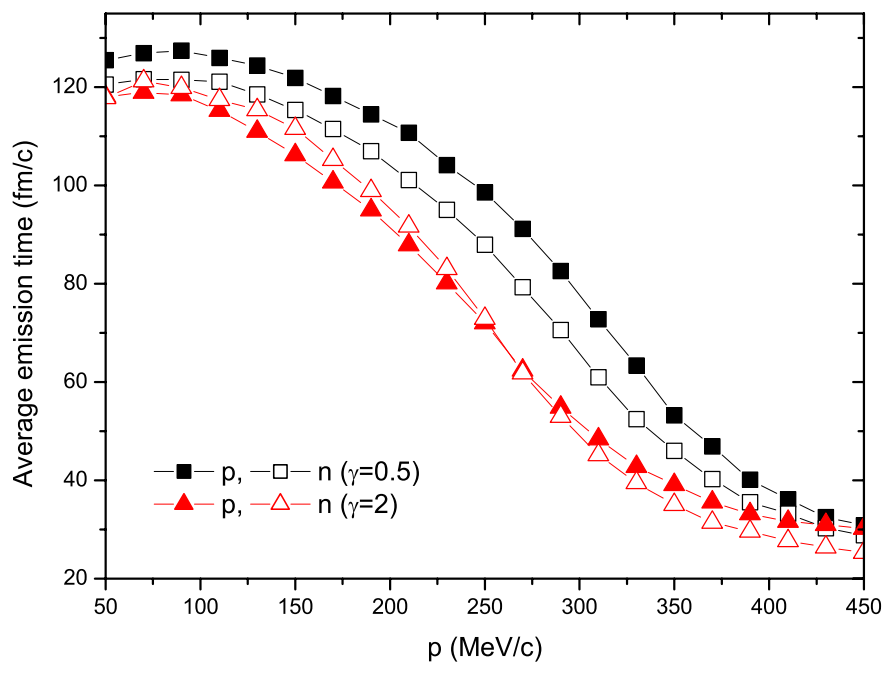

FIG. 8. (Color online) Average emission times of protons and neutrons as functions of their momenta for different symmetry energies.

slightly if the stiff symmetry energy is used for the case of $K_{0}=380 \mathrm{MeV}$ and free $N N$ cross sections (dotted curve). Also shown in Fig. 7 is the time evolution of average central density by using $K_{0}=380 \mathrm{MeV}$ and the soft symmetry energy, but with in-medium $N N$ cross sections $\sigma_{\text {in-medium }}$ (dashdotted curve). One finds that different $N N$ cross sections lead to almost similar time evolutions of average central density. Although the free $N N$ cross sections, which have larger values than the in-medium ones, would lead to more stopping and thus higher density, enhanced Pauli blocking and meanfield pressure effects bring the resulting maximum density similar to that obtained using the in-medium cross sections. As a result, the two different cross sections lead to similar pressures during the high density stage of heavy-ion collisions, and thus give comparable nucleon emission times as shown in Fig. 5(c).

Energies of emitted nucleons are correlated to the times at which they are emitted. Generally, earlier emitted nucleons have higher energies than later emitted ones. This can be seen in Fig. 8, where we show the average emission times of protons and neutrons as functions of their momenta by using $K_{0}=380 \mathrm{MeV}$ with the soft or stiff symmetry energy. Indeed, high momentum nucleons are emitted in the early preequilibrium stage of collisions when the average density is relatively high, while low momentum ones are mainly emitted when the system is close to equilibrium and the average density is low. Figure 8 further shows that the average emission time of nucleons with a given momentum is earlier for the stiff symmetry energy (triangles) than for the soft one (squares). Moreover, there are significant delays in proton emissions (filled squares) with the soft symmetry energy. These features are consistent with the results shown in Fig. 5(a). For stiff symmetry energy, the change in the emission sequence of neutrons and protons with their momenta is consistent with the change in the sign of stiff symmetry potential at low densities as shown in Fig. 1.

We note, however, that the distribution of the emission times for nucleons with same momentum is quite broad par- 


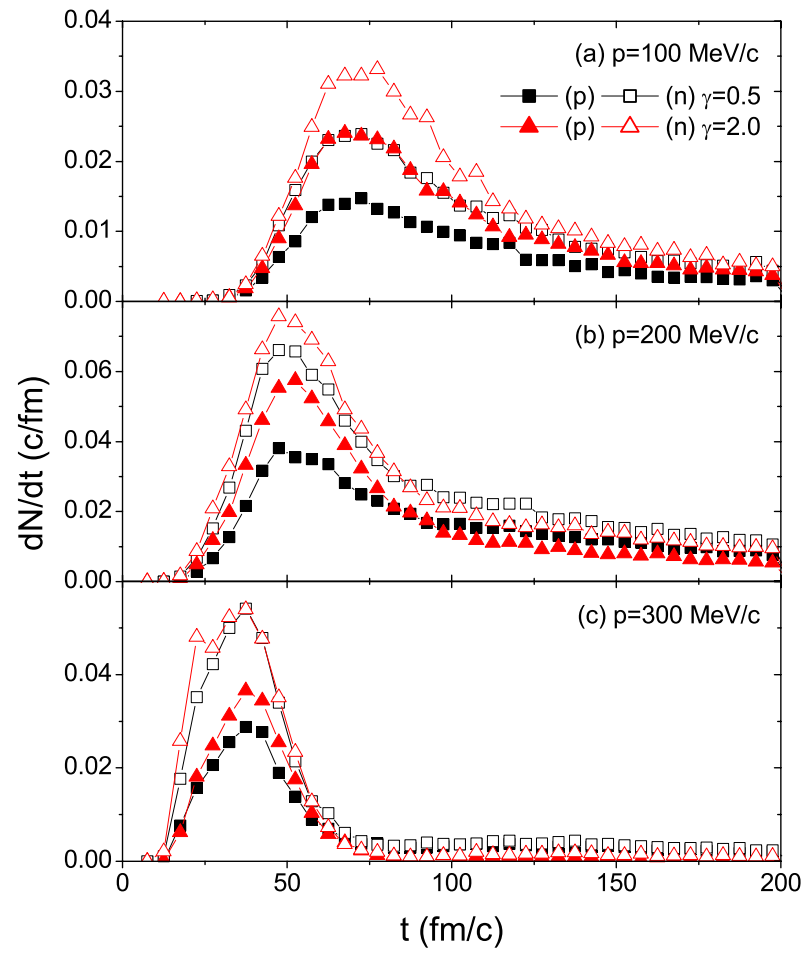

FIG. 9. (Color online) Emission rates of neutrons and protons with momenta (a) 100, (b) 200, and (c) $300 \mathrm{MeV} / c$ (with bin size of $20 \mathrm{MeV} / c$ ) for $K_{0}=380 \mathrm{MeV}$, free $N N$ cross sections, and either soft or stiff symmetry energy.

ticularly for low momentum nucleons. This is shown in Fig. 9 for nucleons with momenta of (a) 100, (b) 200, and (c) $300 \mathrm{MeV} / c$ (all with bin size of $20 \mathrm{MeV} / c$ ), obtained with $K_{0}=380 \mathrm{MeV}$, free $N N$ cross sections, and either soft or stiff symmetry energy. Although the peak emission time increases with decreasing nucleon momentum, the distribution of nucleon emission times is broad. Furthermore, at later times more nucleons with momentum of $200 \mathrm{MeV} / c$ are emitted than those with momentum of $100 \mathrm{MeV} / c$ are emitted. For both protons and neutrons, more are emitted for the stiff than for the soft symmetry energy.

In low and intermediate energy heavy-ion collisions induced by heavy nuclei, the Coulomb interaction is expected to affect the emission time of protons, and thus also the neutron-proton correlation function $[61,62]$. In present IBUU simulations, the Coulomb potential is included explicitly in the dynamic evolution of colliding nuclei. Effects of Coulomb interaction on the proton emission time can be seen in Fig. 10, where the average emission time of protons is shown as a function of their momenta with (filled squares) and without (open squares) Coulomb interaction by using either the soft (left panel) or stiff (right panel) symmetry energy. One finds that the Coulomb interaction is not important for ${ }^{52} \mathrm{Ca}+{ }^{48} \mathrm{Ca}$ as it only shortens the proton average emission time by factors less than $10 \%$ for both the soft and stiff symmetry energies. The Coulomb potential effect is even smaller for protons with high momentum, which are emitted early during collisions. We note that the Coulomb potential does not affect the neutron emission time as expected.

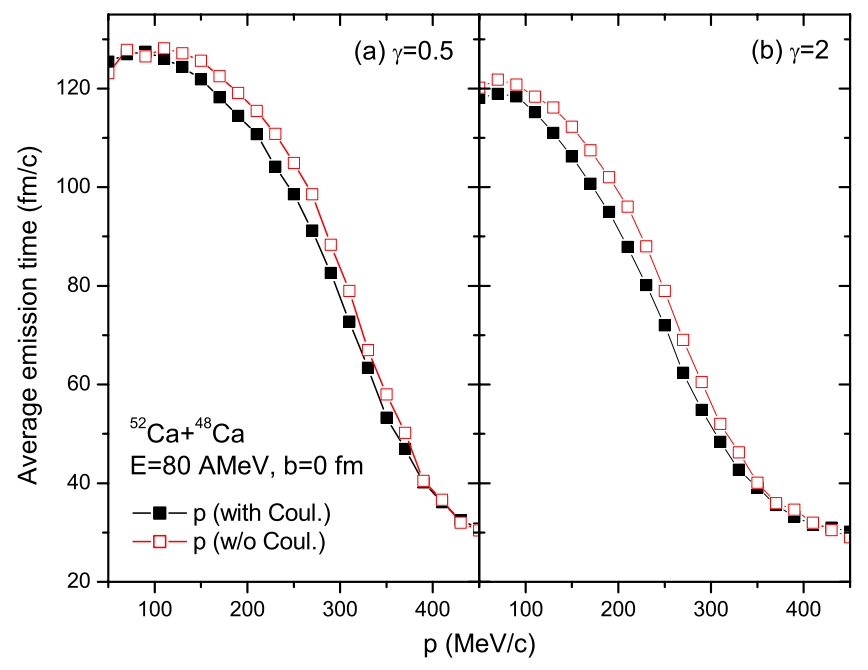

FIG. 10. (Color online) Average emission time of protons as a function of their momenta with (filled squares) and without (open squares) Coulomb interaction by using the soft (a) or stiff (b) symmetry energy.

\section{B. Size of nucleon emission source}

The size of emission source can be characterized by the root-mean-squared radii of emitted protons and neutrons in the transverse $\left(\left\langle R_{\mathrm{rms}}\right\rangle\right)$ and longitudinal $\left(\left\langle Z_{\mathrm{rms}}\right\rangle\right)$ directions. In Fig. 11, we show these radii as functions of nucleon momentum using $K_{0}=380 \mathrm{MeV}$ and free $N N$ cross sections, but with either soft (squares) or stiff (triangles) symmetry energy. It is seen that for both symmetry energies the emission source has an oblate shape with a larger transverse radius than longitudinal radius. Furthermore, the emission source size is larger for the soft than the stiff symmetry energy. However, the difference between the transverse radii of proton and neutron emission sources is more appreciable in

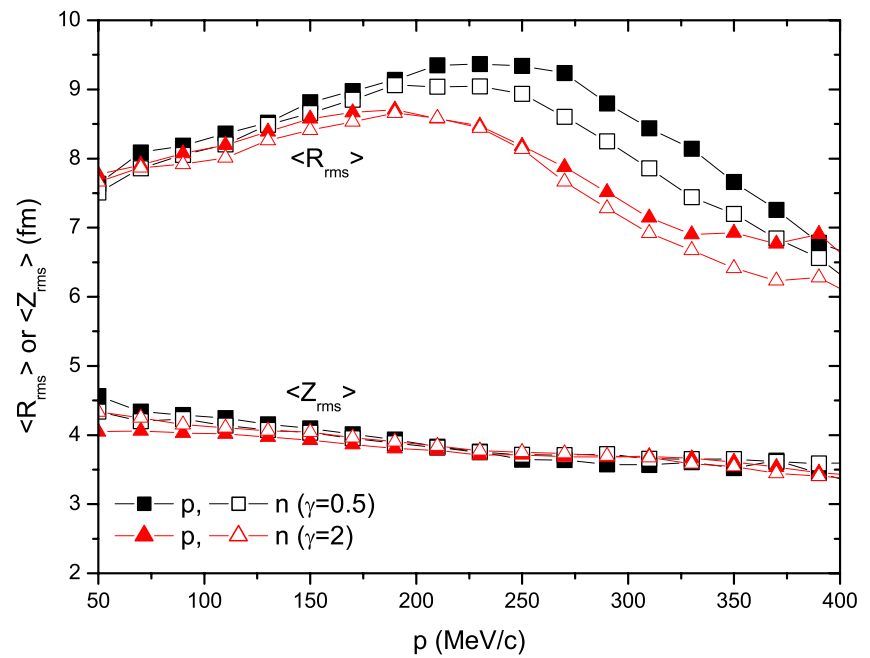

FIG. 11. (Color online) Root-mean-squared transverse emission radius $\left\langle R_{\mathrm{rms}}\right\rangle$ and longitudinal emission radius $\left\langle Z_{\mathrm{rms}}\right\rangle$ of neutrons and protons as functions of their momenta by using $K_{0}$ $=380 \mathrm{MeV}$ with either soft (squares) or stiff (triangles) symmetry energy. 


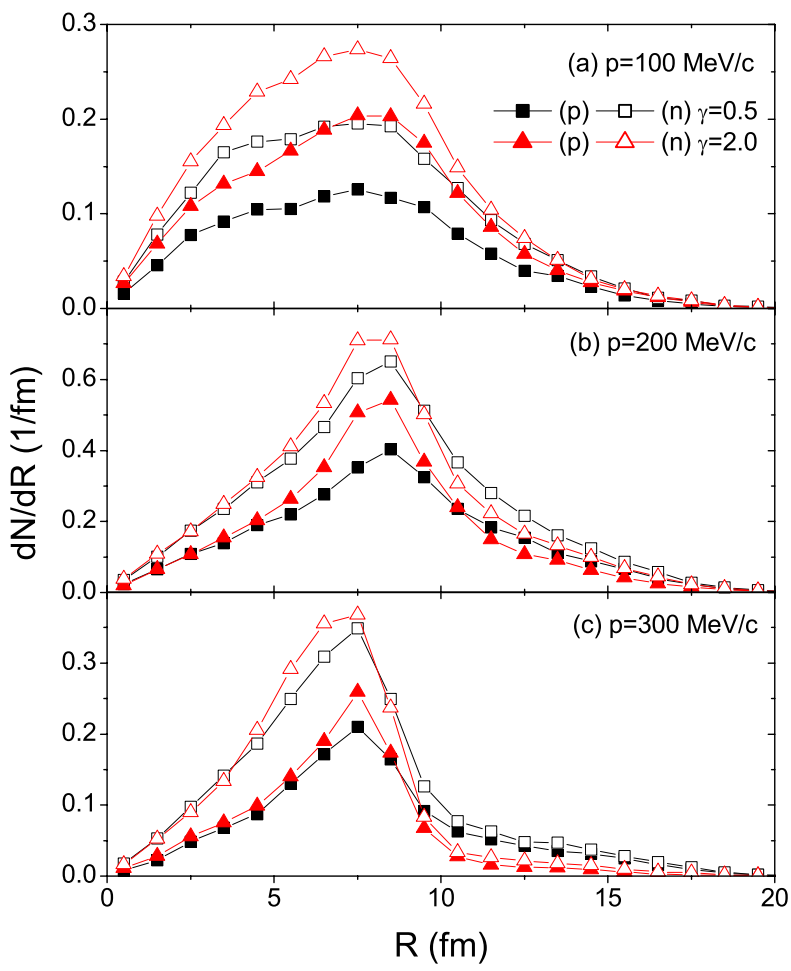

FIG. 12. (Color online) Transverse radius distribution of emitted nucleons with momenta (a) 100, (b) 200, and (c) $300 \mathrm{MeV} / c$ (with bin size of $20 \mathrm{MeV} / c$ ) for $K_{0}=380 \mathrm{MeV}$, free $N N$ cross sections, and either soft or stiff symmetry energy.

the case of soft symmetry energy than stiff symmetry energy. These features are consistent with the emission times shown in Fig. 8.

The decrease of the transverse radius with increasing nucleon momentum shown in Fig. 11 is expected as higher momentum nucleons are emitted earlier when the source size is more compact. The small transverse radius of the emission source for lower momentum nucleons are, on the other hand, due to the broad distributions in nucleon emission times, resulting in the emissions of more intermediate momentum nucleons than low momentum nucleons at later times. As a result, the source size for low momentum nucleons is largely determined by earlier emitted nucleons. This can also been seen in Fig. 12 where we show the transverse radius distributions of emitted nucleons with momenta (a) 100, (b) 200, and (c) $300 \mathrm{MeV} / c$ (with bin size of $20 \mathrm{MeV} / c$ ) for $K_{0}$ $=380 \mathrm{MeV}$, free $N N$ cross sections, and either soft or stiff symmetry energy. It is seen that the peak of the transverse radius distribution first increases then decreases as the nucleon momentum gets larger, consistent with that shown in Fig. 11. Furthermore, the width of the radius distribution is narrower for high momentum nucleons than for low momentum ones.

To see more clearly the size and structure of nucleon emission source, we show in Figs. 13 and 14 the density contours $\rho(x, 0, z)$ in the reaction plane and $\rho(x, y, 0)$ perpendicular to the reaction plane at different times by using $K_{0}$ $=380 \mathrm{MeV}$ and free $N N$ cross sections with the soft symmetry energy. It is seen that after initial contact of the target and projectile at $1 \mathrm{fm} / c$, the nuclear matter is compressed to high density during the first $20 \mathrm{fm} / c$. This is followed by expansions, mainly in the transverse direction, leading to the formation of an oblate bubble nuclear matter, which subsequently changes into a ring-shaped structure. The ring structure has a long lifetime and decays into several fragments after about $100 \mathrm{fm} / c$. Such exotic structures also exist in the other cases, i.e., using $K_{0}=201 \mathrm{MeV}$, stiff symmetry energy, or in-medium $N N$ cross sections. This interesting phenom-

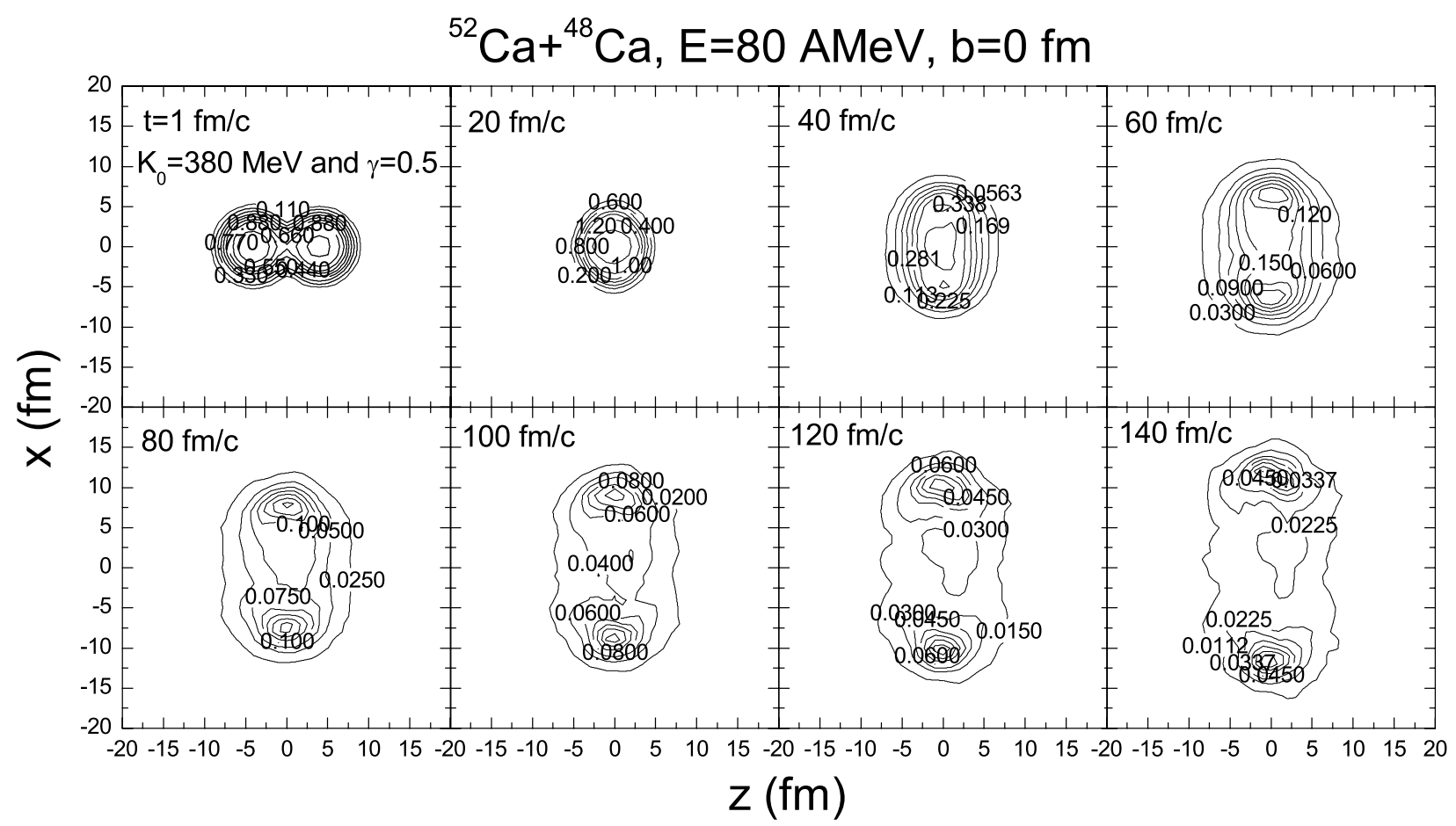

FIG. 13. Density contours $\rho(x, 0, z)$ at different times by using $K_{0}=380 \mathrm{MeV}$ and free $N N$ cross sections with the soft symmetry energy. 


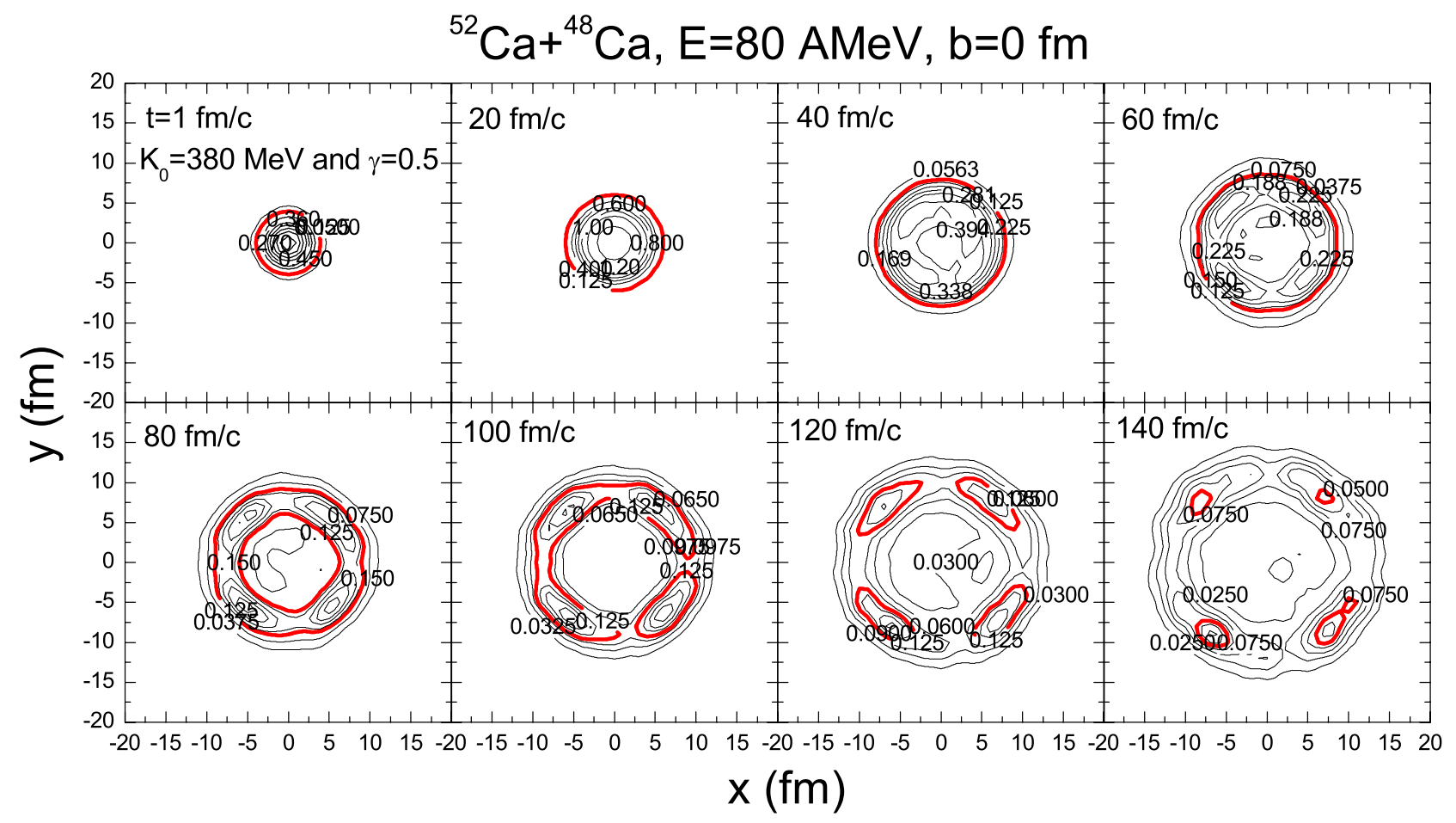

FIG. 14. (Color online) Density contours $\rho(x, y, 0)$ at different times by using $K_{0}=380 \mathrm{MeV}$ and free $N N$ cross sections with the soft symmetry energy. The thick curves represent $\rho_{0} / 8$.

enon is not new and was studied extensively a decade ago. For medium mass nuclei, this is a common phenomenon in central or near central collisions at energy considered here [63-65]. The formation of bubble and ring structures during expansion implies that the central density shown in Fig. 7 is not always the maximum density reached in collisions. In Fig. 15, we show the time evolution of maximum density for different incompressibilities, $N N$ cross sections, and symmetry energies as in Fig. 7. We see that after about $30 \mathrm{fm} / c$ when the bubble is formed, the maximum density has a value

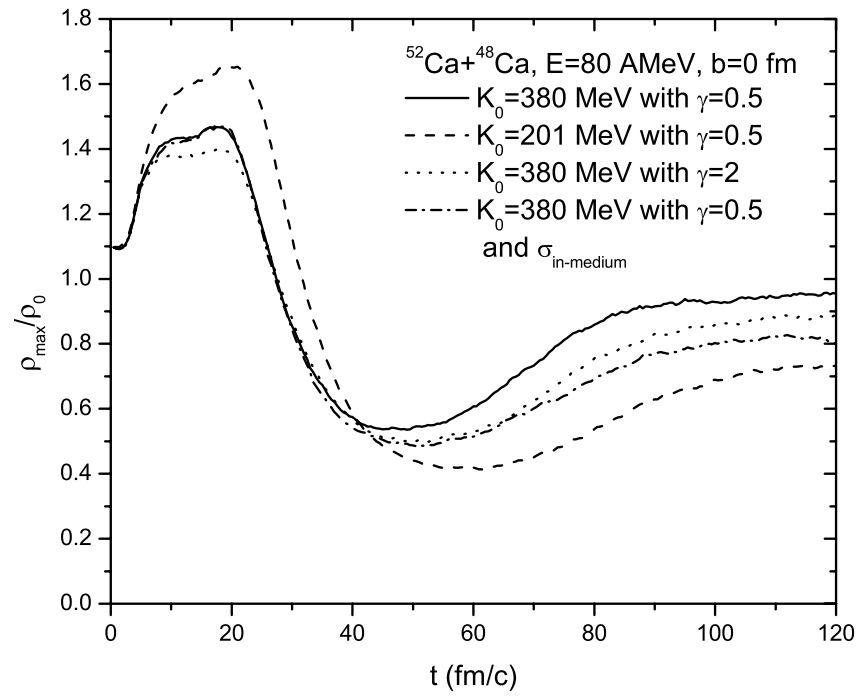

FIG. 15. Time evolution of maximum density for different incompressibilities, $N N$ cross sections, and symmetry energies as in Fig. 7. around normal nuclear density and is very different from the central density shown in Fig. 7. Since low momentum nucleons are mainly emitted during the late stage of heavy-ion collisions from the ring and its decaying fragments, the symmetry energy effect is thus small as a result of the small difference between soft and stiff symmetry potentials at normal nuclear density as shown in Fig. 1.

\section{Momentum distributions of emitted nucleons}

Figure 16 shows the momentum distributions of emitted neutrons and protons for $K_{0}=380 \mathrm{MeV}$ and free $N N$ cross

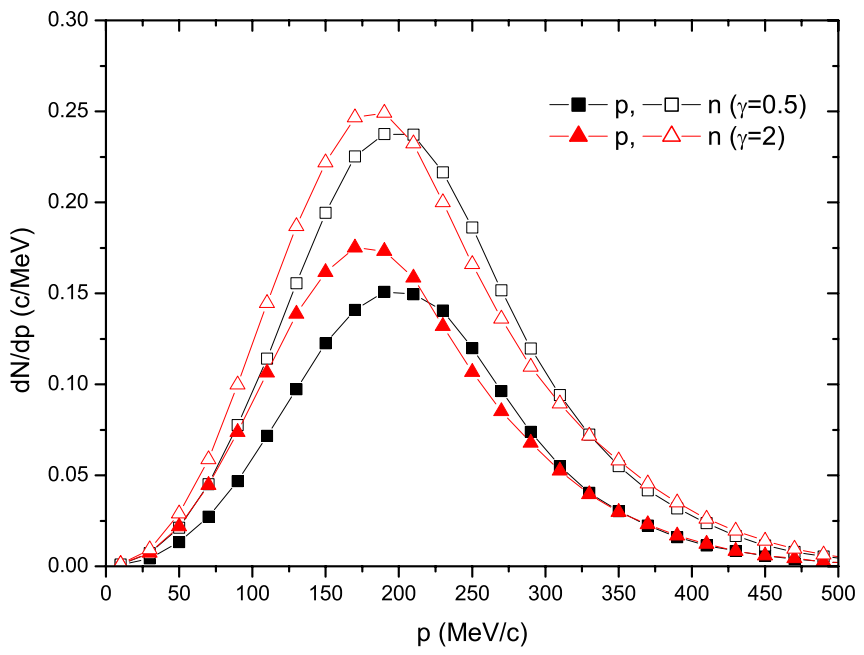

FIG. 16. (Color online) Momentum distributions of emitted neutrons and protons for $K_{0}=380 \mathrm{MeV}$ and free $N N$ cross sections with soft (squares) or stiff (triangles) symmetry energy. 
sections with soft (squares) or stiff (triangles) symmetry energy. It is seen that most nucleons are emitted with momenta around $p=180 \mathrm{MeV} / c$, and the peak momentum shifts to a larger value as the symmetry energy becomes softer. The lower peak momentum in the case of stiff symmetry energy is due to the larger potential energy during initial compressions, and thus lower average nucleon kinetic energy as a result of energy conservation. Furthermore, symmetry energy effects on lower momentum nucleons are stronger for protons than for neutrons, which is consistent with the larger variation of proton symmetry potential at low densities with the stiffness of symmetry energy than that of neutron symmetry potential as shown in Fig. 1 .

\section{NUCLEON-NUCLEON CORRELATION FUNCTIONS}

The nucleon emission function, which is important for understanding the reaction dynamics in heavy-ion collisions, can be extracted from two-particle correlation functions; see, e.g., Refs. [27-30] for reviews. In most studies, only the two-proton correlation function has been measured [6669,59]. Recently, data on two-neutron and neutron-proton correlation functions have also become available. The neutron-proton correlation function is especially useful as it is free of correlations due to wave function antisymmetrization and Coulomb interactions. Indeed, Ghetti et al. have deduced from measured neutron-proton correlation function the time sequence of neutron and proton emissions [70,71].

In the standard Koonin-Pratt formalism [72-74], the twoparticle correlation function is obtained by convoluting the emission function $g(\mathbf{p}, x)$, i.e., the probability for emitting a particle with momentum $\mathbf{p}$ from the space-time point $x$ $=(\mathbf{r}, t)$, with the relative wave function of the two particles, i.e.,

$$
C(\mathbf{P}, \mathbf{q})=\frac{\int d^{4} x_{1} d^{4} x_{2} g\left(\mathbf{P} / 2, x_{1}\right) g\left(\mathbf{P} / 2, x_{2}\right)|\phi(\mathbf{q}, \mathbf{r})|^{2}}{\int d^{4} x_{1} g\left(\mathbf{P} / 2, x_{1}\right) \int d^{4} x_{2} g\left(\mathbf{P} / 2, x_{2}\right)} .
$$

In the above, $\mathbf{P}\left(=\mathbf{p}_{1}+\mathbf{p}_{2}\right)$ and $\mathbf{q}\left[=(1 / 2)\left(\mathbf{p}_{1}-\mathbf{p}_{2}\right)\right]$ are, respectively, the total and relative momenta of the particle pair; and $\phi(\mathbf{q}, \mathbf{r})$ is the relative two-particle wave function, with $\mathbf{r}$ being their relative position, i.e., $\mathbf{r}=\left(\mathbf{r}_{2}-\mathbf{r}_{1}\right)-(1 / 2)\left(\mathbf{v}_{1}\right.$ $\left.+\mathbf{v}_{2}\right)\left(t_{2}-t_{1}\right)$. This approach has been very useful in studying effects of nuclear equation of state and nucleon-nucleon cross sections on the reaction dynamics of intermediate energy heavy-ion collisions [28]. In the present paper, we use the Koonin-Pratt method to determine the nucleon-nucleon correlation functions in order to study the effect due to the density dependence of nuclear symmetry energy on the spatial and temporal structure of nucleon emission source in intermediate energy heavy-ion collisions.

\section{A. Symmetry energy effects}

Using the program Correlation After Burner [75], which takes into account final-state nucleon-nucleon interactions,

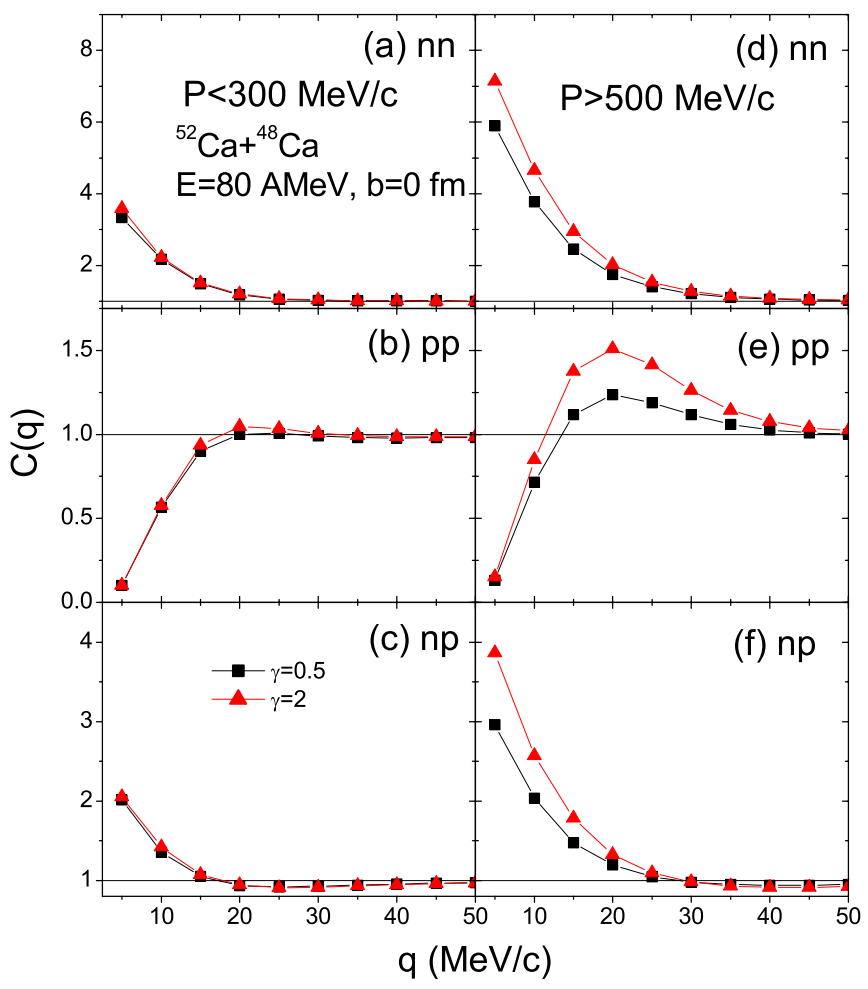

FIG. 17. (Color online) Two-nucleon correlation functions gated on total momentum of nucleon pairs using the soft (filled squares) or stiff (filled triangles) symmetry energy. Left panels are for $P$ $<300 \mathrm{MeV} / c$ while the right panels are for $P>500 \mathrm{MeV} / c$.

we have evaluated two-nucleon correlation functions from the emission function given by the IBUU model. Shown in Fig. 17 are two-nucleon correlation functions gated on total momentum $P$ of nucleon pairs from central collisions of ${ }^{52} \mathrm{Ca}+{ }^{48} \mathrm{Ca}$ at $E=80 \mathrm{MeV} /$ nucleon. The left and right panels are for $P<300 \mathrm{MeV} / c$ and $P>500 \mathrm{MeV} / c$, respectively. For both neutron-neutron (upper panels) and neutron-proton (lower panels) correlation functions, they peak at $q$ $\approx 0 \mathrm{MeV} / c$. The proton-proton correlation function (middle panel) is, however, peaked at about $q=20 \mathrm{MeV} / c$ due to strong final-state $s$-wave attraction, but is suppressed at $q$ $=0$ as a result of Coulomb repulsion and antisymmetrization of two-proton wave function. These general features are consistent with those observed in experimental data from heavyion collisions [70].

Since emission times of low-momentum nucleons do not change much with the different $E_{\text {sym }}(\rho)$ used in the IBUU model as shown in Fig. 8, two-nucleon correlation functions are not much affected by the stiffness of symmetry energy. On the other hand, the emission times of high-momentum nucleons, which are dominated by those with momenta near $250 \mathrm{MeV} / c$, differ appreciably for the two symmetry energies considered here. Correlation functions of highmomentum nucleon pairs thus show a strong dependence on the nuclear symmetry energy. Gating on nucleon pairs with high total momentum allows one to select those nucleons that have short average spatial separations at emissions, and thus exhibit enhanced correlations. For these nucleon pairs with high total momentum, strength of their correlation func- 


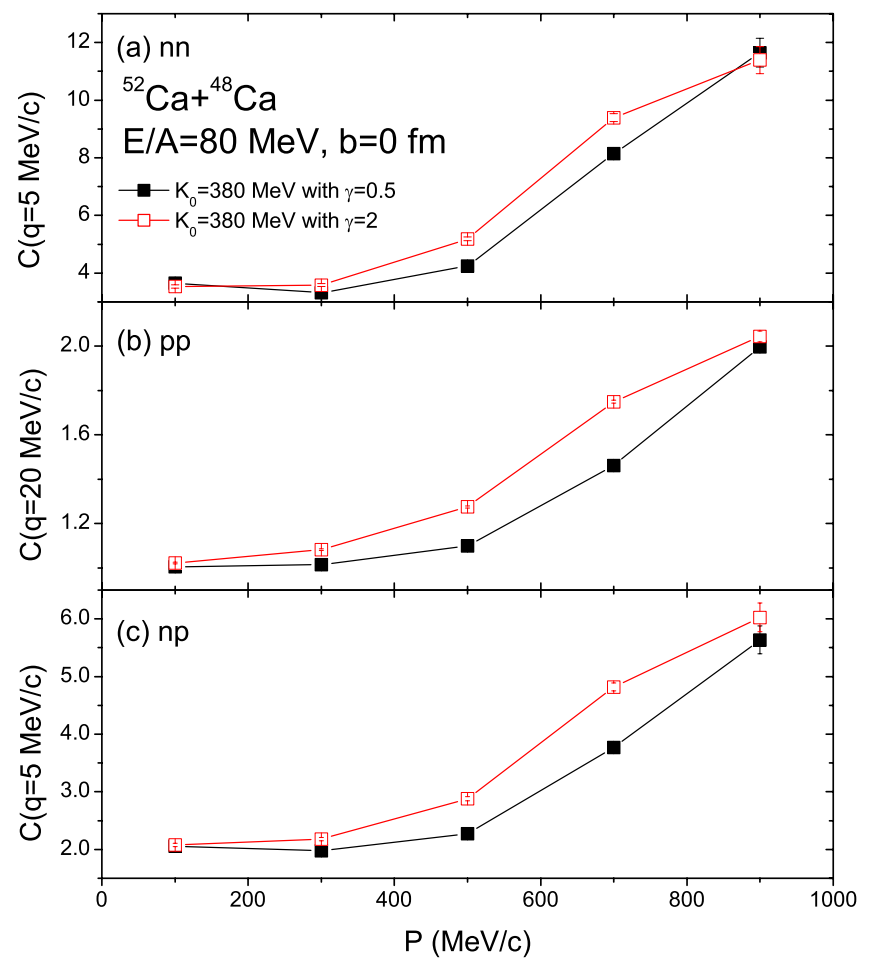

FIG. 18. (Color online) Total momentum dependence of the correlation functions for neutron-neutron (a) and neutron-proton (c) pairs with relative momentum $q=5 \mathrm{MeV} / c$ and proton-proton (b) pairs with relative momentum $q=20 \mathrm{MeV} / c$ by using $K_{0}$ $=380 \mathrm{MeV}$ with both soft (filled squares) and stiff (open squares) symmetry energies.

tion is stronger for the stiff symmetry energy than for the soft symmetry energy: about $30 \%$ and $20 \%$ for neutron-proton pairs and neutron-neutron pairs at low relative momentum $q=5 \mathrm{MeV} / c$, respectively, and $20 \%$ for proton-proton pairs at $q=20 \mathrm{MeV} / c$. The neutron-proton correlation function thus exhibits the highest sensitivity to variations of $E_{\text {sym }}(\rho)$. As shown in Fig. 8 and discussed earlier, the emission sequence of neutrons and protons is sensitive to $E_{\text {sym }}(\rho)$. With stiff $E_{\text {sym }}(\rho)$, high-momentum neutrons and protons are emitted almost simultaneously, and they are thus temporally strongly correlated, leading to a larger neutron-proton correlation function. On the other hand, proton emissions in the case of soft $E_{\text {sym }}(\rho)$ are delayed compared to neutrons, so they are temporally weakly correlated with neutrons. The resulting values of neutron-proton correlation function are thus smaller. Furthermore, both neutrons and protons are emitted earlier with stiff $E_{\text {sym }}(\rho)$, so they have shorter spatial separation at emissions. As a result, neutrons and protons are more correlated for the stiff symmetry energy than for the soft symmetry energy. Our results thus clearly demonstrate that correlation functions of nucleon pairs with high total momentum can indeed reveal sensitively the effect of nuclear symmetry energy on the temporal and spatial distributions of emitted nucleons.

To see more clearly the symmetry energy effect on nucleon-nucleon correlation functions, we show in Fig. 18 the total momentum dependence of the correlation functions for neutron-neutron and neutron-proton pairs with relative momentum $q=5 \mathrm{MeV} / c$ and proton-proton pairs with relative momentum $q=20 \mathrm{MeV} / c$ by using $K_{0}=380 \mathrm{MeV}$ with both soft (filled squares) and stiff (open squares) symmetry energies. It is seen that the symmetry energy effect is more pronounced for pairs with higher total momentum as discussed previously. However, the effect becomes weaker when their total momentum becomes very large. This is due to the fact that very energetic neutrons and protons mainly come from very early stage of collisions when the reaction system reaches maximum compression and nucleon emissions are largely affected by the isospin-independent part of EOS. The symmetry energy effect on the emission time of nucleons is therefore reduced for these very energetic neutrons and protons as shown in Fig. 8. Furthermore, emission times of very energetic neutrons and protons are strongly influenced by the high density behavior of symmetry energy, and for the stiff symmetry energy very high momentum neutrons are emitted earlier than protons as shown in Fig. 8. As a result, the symmetry energy effect on the correlation function of very energetic neutron-proton pairs is also reduced.

Since the nuclear symmetry energy affects differently the transverse and longitudinal radii of nucleon emission source, it is useful to study also the dependence of two-nucleon correlation functions on the direction of emitted nucleons. We have thus considered both transverse cut with $\theta_{\text {c.m. }}=90^{\circ}$ $\pm 10^{\circ}$ and longitudinal cut with $\theta_{\text {c.m. }}=0^{\circ}-20^{\circ}$ or $160^{\circ}-180^{\circ}$, where $\theta_{\text {c.m. }}$ is the center-of-mass angle with respect to the beam direction. Results similar to Fig. 17 for two-proton, two-neutron, and neutron-proton correlation functions using both stiff and soft symmetry energies but stiff isospin-independent EOS and free $N N$ cross sections are shown in Figs. 19 and 20 for transverse and longitudinal cuts, respectively. It is seen that the transverse cut gives smaller values for nucleon-nucleon correlation functions than the longitudinal cut. This is easy to understand as the correlation functions with transverse (longitudinal) cut mainly reflect the transverse (longitudinal) size or lifetime of the emission source. A transverse radius larger than the longitudinal radius shown in Fig. 11 thus leads to smaller values for the nucleon-nucleon correlation functions. Our results further show that the correlation functions of nucleon pairs with total momentum $P>500 \mathrm{MeV} / c$ obtained with transverse cut have stronger symmetry energy dependence than those obtained with longitudinal cut. For transverse cut, the symmetry energy effects are about $23 \%$ and $33 \%$ for neutron-neutron and neutron-proton pairs at $q=5 \mathrm{MeV} / c$, and $22 \%$ for proton-proton pairs at $q=20 \mathrm{MeV} / c$, respectively, but corresponding effects are 14\%, 22\%, and $19 \%$, respectively, for longitudinal cut. However, compared to results in Fig. 17 without the angular cut, there is only a slight enhancement of two-nucleon correlation functions when the transverse cut is imposed, as most nucleons are emitted in the transverse direction.

In the above analysis, the IBUU calculations are terminated at $200 \mathrm{fm} / c$. In Fig. 21, we show the stop time dependence of the correlation functions for high total momentum $(P>500 \mathrm{MeV})$ neutron-neutron and neutron-proton pairs with relative momentum $q=5 \mathrm{MeV} / c$ and proton-proton pairs with relative momentum $q=20 \mathrm{MeV} / c$ by using $K_{0}$ 


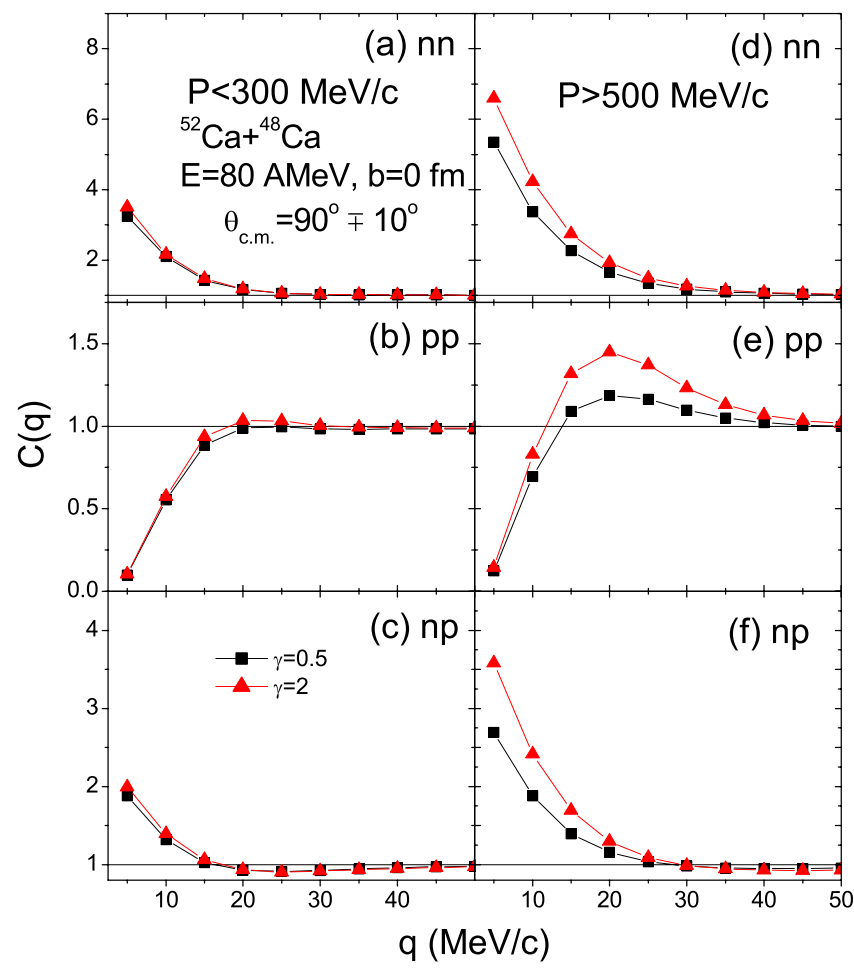

FIG. 19. (Color online) Same as Fig. 17 but with angular cut $\theta_{\text {c.m. }}=90^{\circ} \pm 10^{\circ}$ in center-of-mass (c.m.) system.

$=380 \mathrm{MeV}$ with soft (filled squares) or stiff (open squares) symmetry energy. It is seen that values of these correlation functions are essentially fixed after about $150 \mathrm{fm} / c$ for both soft (open squares) and stiff (filled squares) symmetry ener-

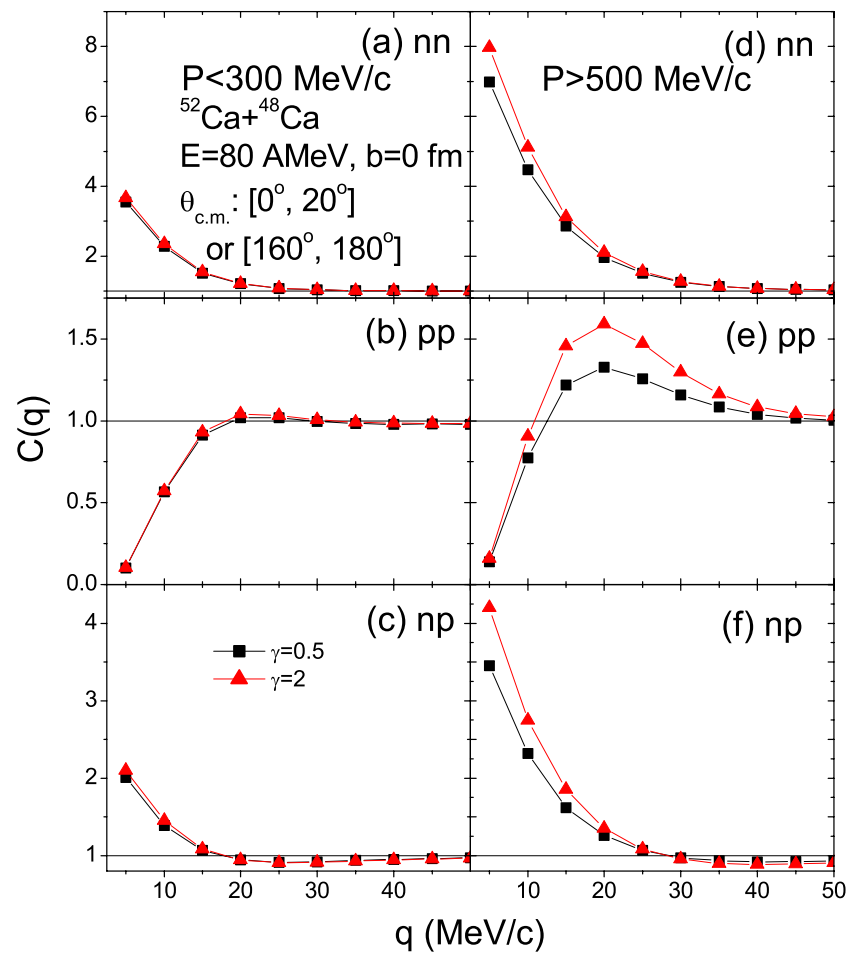

FIG. 20. (Color online) Same as Fig. 17 but with angular cut $\theta_{\text {c.m. }}=0^{\circ}-20^{\circ}$ or $160^{\circ}-180^{\circ}$ in center-of-mass system.

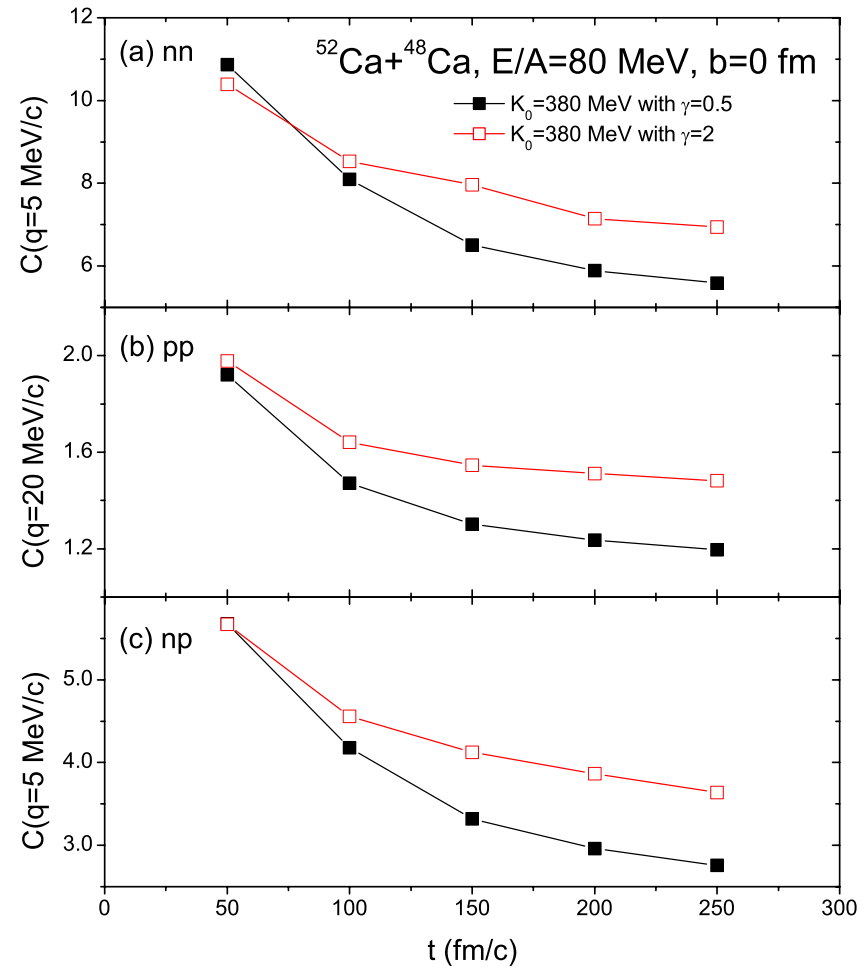

FIG. 21. (Color online) Dependence of two-nucleon correlation functions on stop times for high total momentum $(P>500 \mathrm{MeV})$ neutron-neutron (a) and neutron-proton (c) pairs with relative momentum $q=5 \mathrm{MeV} / c$ and proton-proton (b) pairs with relative momentum $q=20 \mathrm{MeV} / c$ by using $K_{0}=380 \mathrm{MeV}$ with soft (filled squares) or stiff (open squares) symmetry energy.

gies. Furthermore, the symmetry energy effect starts to appear after $50 \mathrm{fm} / c$, when the central density is about $1 / 5 \rho_{0}$ of normal nuclear matter density as shown in Fig. 7 and the maximum density is about $1 / 2 \rho_{0}$ as shown in Fig. 15, indicating that two-nucleon correlation functions are sensitive to the properties of nuclear symmetry energy at densities around and below the normal nuclear density.

\section{B. EOS and $N N$ cross section effects}

We have also studied the effects of isoscalar potential and in-medium $N N$ cross sections on nucleon-nucleon correlation functions. In Fig. 22, we show gated two-nucleon correlation functions from central collisions of ${ }^{52} \mathrm{Ca}+{ }^{48} \mathrm{Ca}$ at $E=80 \mathrm{MeV} /$ nucleon using the soft symmetry energy but different values of incompressibility $K_{0}$ and $N N$ cross sections. One finds that reducing $K_{0}$ from 380 (filled squares) to 201 (filled triangles) $\mathrm{MeV}$ increases the value of the correlation function for high momentum pairs $(P>500 \mathrm{MeV} / c)$ but decreases that for low momentum pairs $(P<300 \mathrm{MeV} / c)$. Specifically, the increases are about $8 \%$ and $13 \%$ for high momentum neutron-proton and neutron-neutron pairs with relative momentum $q=5 \mathrm{MeV} / c$, and about $5 \%$ for high momentum proton-proton pairs with relative momentum $q$ $=20 \mathrm{MeV} / c$, while corresponding decreases for low momentum pairs are about $14 \%, 3 \%$, and $12 \%$. Changing experimental free-space $N N$ cross section $\sigma_{\exp }$ to in-medium $N N$ cross sections $\sigma_{\text {in-medium }}$ (open triangles) leads to 


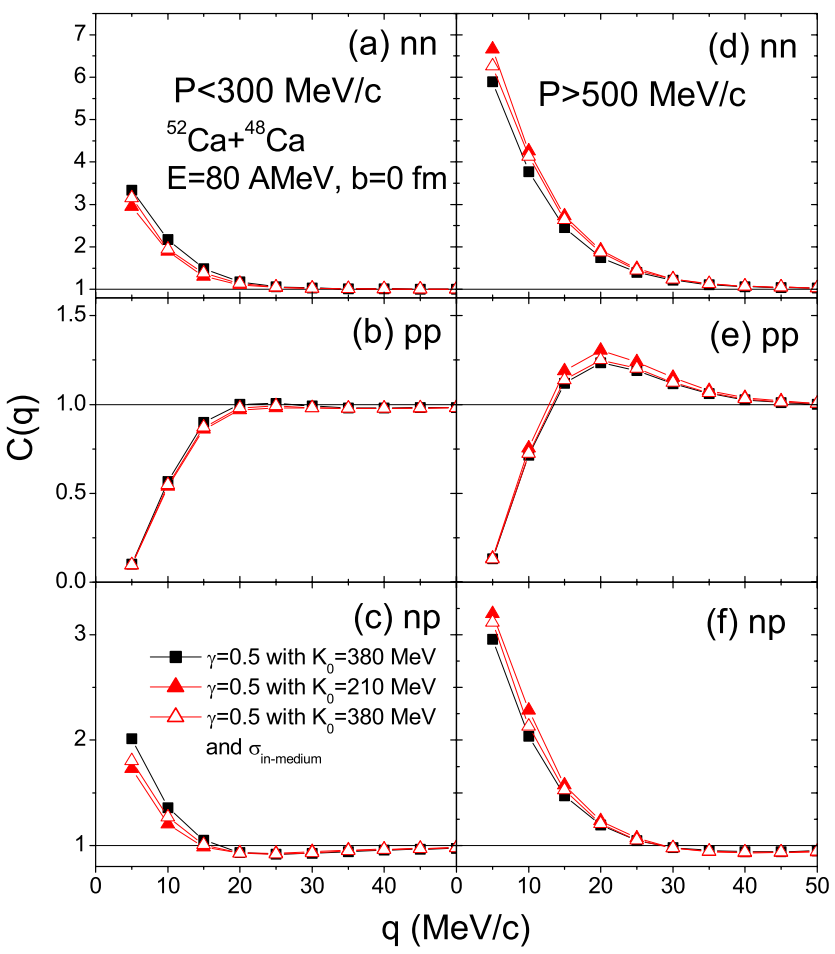

FIG. 22. (Color online) Two-nucleon correlation functions gated on total momentum of nucleon pairs using the soft symmetry energy but different values of incompressibility $K_{0}$ and $N N$ cross sections. Left panels are for $P<300 \mathrm{MeV} / c$ while right panels are for $P>500 \mathrm{MeV} / c$.

changes of about $5 \%, 6 \%$, and $1 \%$ in the neutron-proton, neutron-neutron, and proton-proton correlation functions for high momentum particle pairs $(P>500 \mathrm{MeV} / c)$, but about $10 \%, 2 \%$, and $5 \%$ for low momentum particle pairs $(P$ $<300 \mathrm{MeV} / c$ ). These results are similar to those found in Ref. [28]. The weak dependence of two-nucleon correlation functions on the stiffness of isoscalar potential and the $N N$ cross sections is consistent with the nucleon emission times shown in the last section.

\section{Impact parameter and incident energy dependence}

The nucleon-nucleon correlation functions also depend on the impact parameter and incident energy of heavy-ion collisions. Figure 23 shows the impact parameter dependence of neutron-neutron and neutron-proton correlation functions at a relative momentum $q=5 \mathrm{MeV} / c$ and proton-proton correlation function at a relative momentum $q=20 \mathrm{MeV} / c$ for particle pairs with high total momentum $P>500 \mathrm{MeV} / c$ by using $K_{0}=380 \mathrm{MeV}$ and free $N N$ cross sections with the soft (filled squares) or stiff (open squares) symmetry energy. It is seen that the symmetry energy effect is stronger in central and semicentral collisions and becomes weaker in semiperipheral and peripheral collisions. This simply reflects the fact that nuclear compression is weaker in peripheral collisions, and the symmetry energy effect mainly comes from the small difference between the soft and stiff symmetry energies at low densities. Also, one finds that values of these correlation functions decrease with increasing impact param-

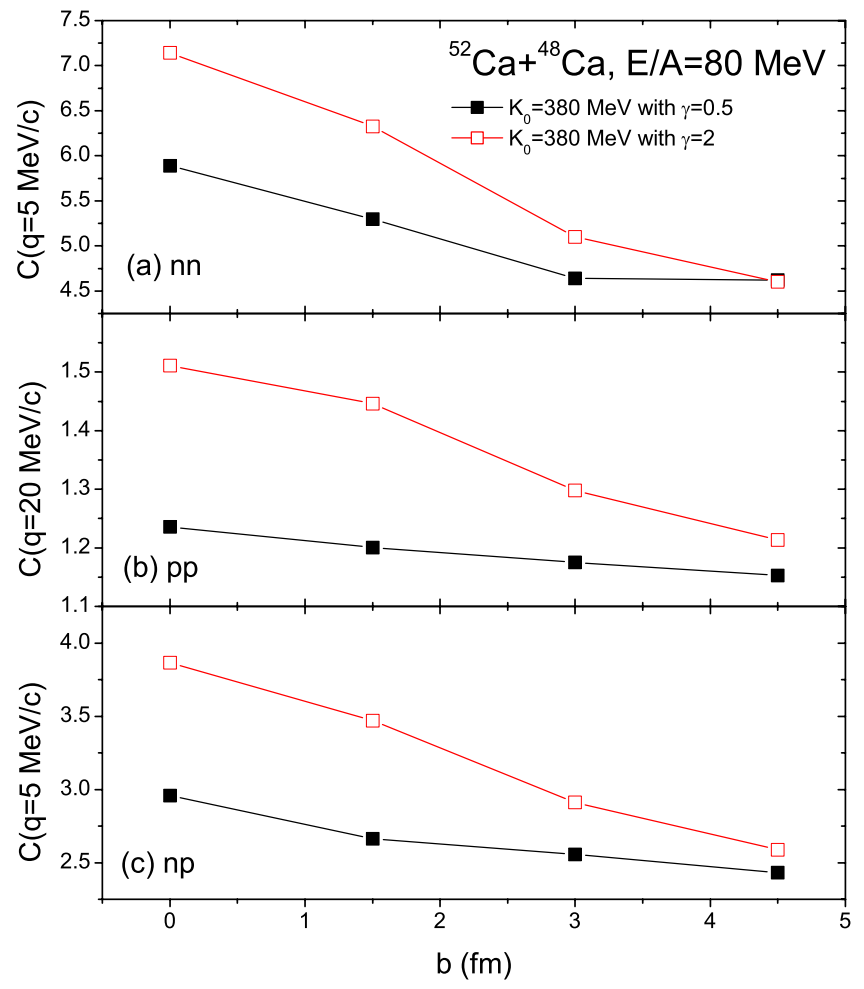

FIG. 23. (Color online) Impact parameter dependence of neutron-neutron (a) and neutron-proton (c) correlation functions at relative momentum $q=5 \mathrm{MeV} / c$ and proton-proton (b) correlation function at relative momentum $q=20 \mathrm{MeV} / c$ for particle pairs with high total momentum $P>500 \mathrm{MeV} / c$ by using $K_{0}=380 \mathrm{MeV}$ and free $N N$ cross sections with the soft (filled squares) or stiff (open squares) symmetry energy.

eter, which is consistent with previous BUU results for the proton-proton correlation function [67]. The correlation function obtained with the stiff symmetry energy exhibits, however, a stronger dependence on impact parameter than that using the soft symmetry energy as the former has a stronger density dependence.

Dependence of nucleon-nucleon correlation functions on the incident energy of heavy-ion collisions is shown in Fig. 24 for high total momentum $(P>500 \mathrm{MeV})$ neutron-neutron and neutron-proton pairs with relative momentum $q$ $=5 \mathrm{MeV} / c$ and for proton-proton pairs with relative momentum $q=20 \mathrm{MeV} / c$. The results are obtained using $K_{0}$ $=380 \mathrm{MeV}$ and free $N N$ cross sections with the soft (filled squares) or stiff (open squares) symmetry energy. It is seen that the values of nucleon-nucleon correlation functions increase with increasing incident energy. This is understandable since nuclear compression increases with increasing incident energy, and nucleons are also emitted earlier, leading to a smaller source size. Also, the stiff symmetry energy gives a larger value for the nucleon-nucleon correlation function than the soft symmetry energy, and the difference does not depend much on incident energies, although the relative effect is reduced. This is due to the fact that at high incident energies the symmetry energy effect mainly comes from early compression stage of collisions when nuclear pressure affects strongly the emission times of nucleons. Furthermore, 


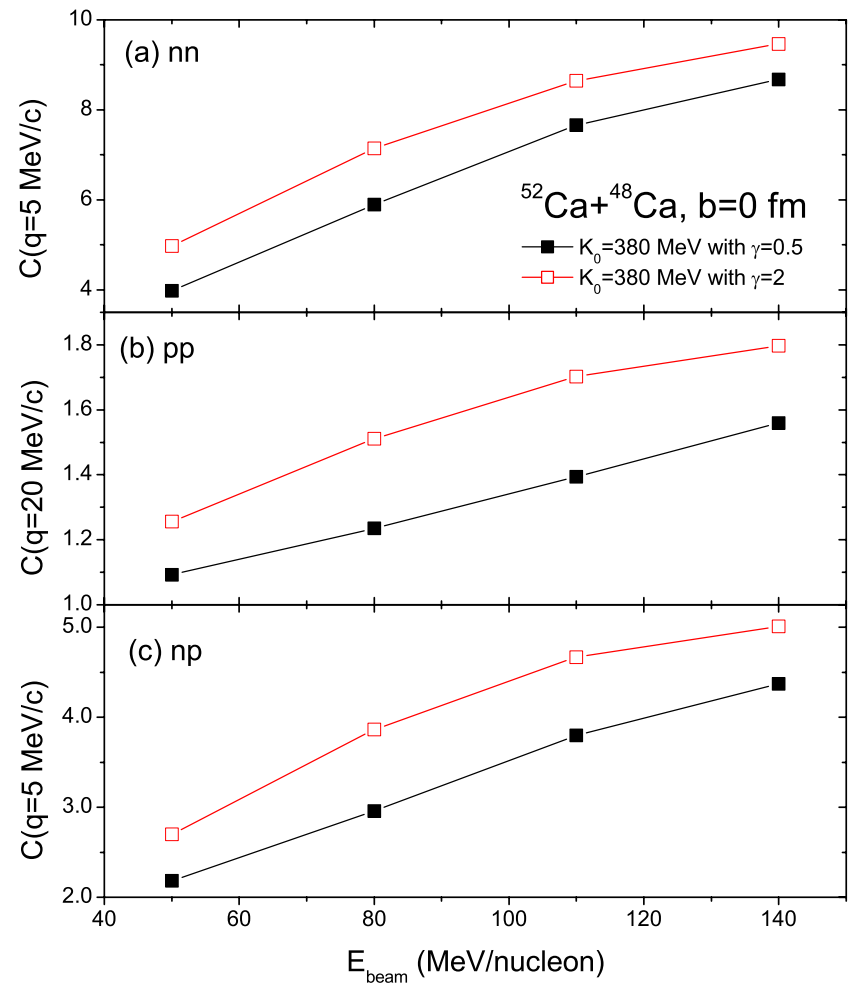

FIG. 24. (Color online) Dependence of nucleon-nucleon correlation functions on the incident energy for hight total momentum $(P>500 \mathrm{MeV})$ neutron-neutron (a) and neutron-proton (c) pairs with relative momentum $q=5 \mathrm{MeV} / c$ and proton-proton (b) pairs with relative momentum $q=20 \mathrm{MeV} / c$ by using $K_{0}=380 \mathrm{MeV}$ and free $N N$ cross sections with the soft (filled squares) or stiff (open circles) symmetry energy.

the system expands rapidly at high incident energies, the low density behavior of symmetry potential thus plays a less important role on the relative emission time of neutrons and protons. According to Fig. 8, nucleons are emitted earlier with stiff rather than with soft symmetry energies. Therefore, the symmetry potential effects remain appreciable with increasing incident energy. On the other hand, the initial compression is relatively weak at lower incident energies, and the behavior of symmetry energy at low densities becomes relevant.

\section{Reaction system dependence}

To see how the above results change for different reaction systems, we show in Fig. 25 two-nucleon correlation functions for head-on collisions of ${ }^{132} \mathrm{Sn}+{ }^{124} \mathrm{Sn}$ at $E$ $=80 \mathrm{MeV} /$ nucleon by using the soft (squares) or stiff (triangles) symmetry energy. This reaction system has a similar isospin asymmetry, i.e., $\delta=0.22$ as the reaction system ${ }^{52} \mathrm{Ca}+{ }^{48} \mathrm{Ca}$ studied in the above, and can also be studied in future RIA. It is seen that two-nucleon correlation functions in this case are similar to those in ${ }^{52} \mathrm{Ca}+{ }^{48} \mathrm{Ca}$ reactions shown in Fig. 17. The strength of two-nucleon correlation functions at small relative momenta is, however, about a factor of 2 smaller than that in the reaction system ${ }^{52} \mathrm{Ca}$ $+{ }^{48} \mathrm{Ca}$. This reduction is mainly due to the larger size of the

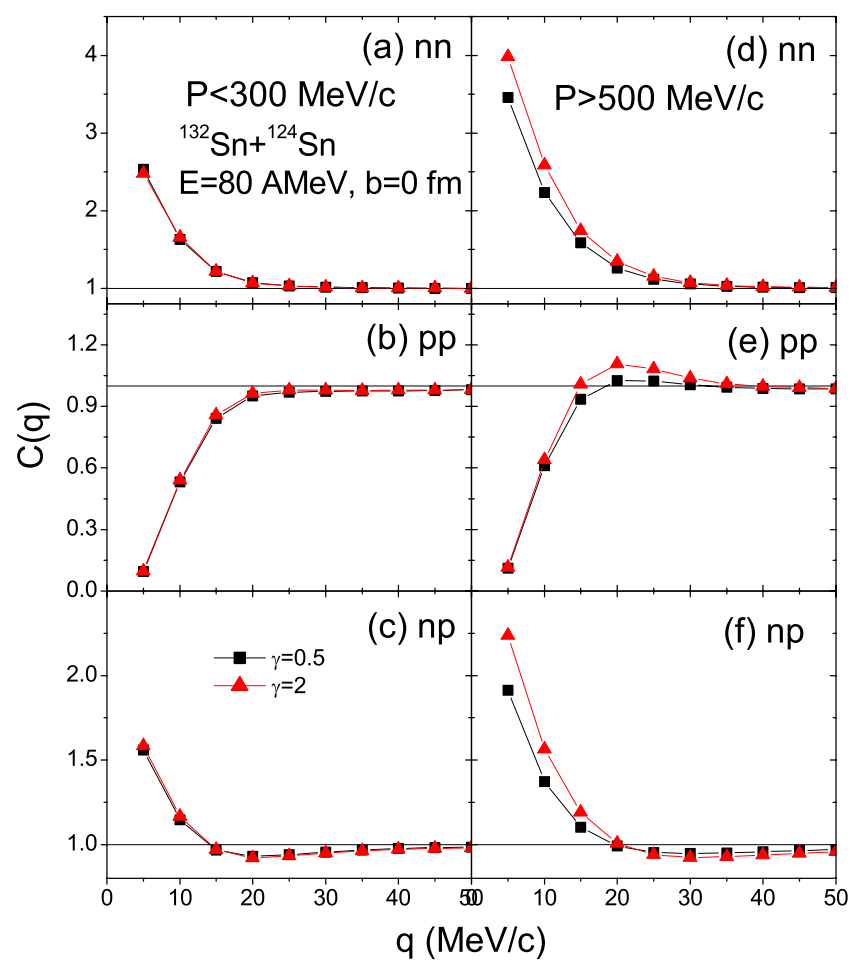

FIG. 25. (Color online) Two-nucleon correlation functions gated on total momentum of nucleon pairs using the soft (filled squares) or stiff (filled triangles) symmetry energy for the central reaction system ${ }^{132} \mathrm{Sn}+{ }^{124} \mathrm{Sn}$ at $E=80 \mathrm{MeV} /$ nucleon. Left panels are for $P<300 \mathrm{MeV} / c$ while right panels are for $P>500 \mathrm{MeV} / c$.

reaction system ${ }^{132} \mathrm{Sn}+{ }^{124} \mathrm{Sn}$ than ${ }^{52} \mathrm{Ca}+{ }^{48} \mathrm{Ca}$. Because of stronger Coulomb repulsion for protons, the symmetry energy effect on the correlation functions of neutron-proton pairs and proton-proton pairs is weaker compared with those in the reaction system ${ }^{52} \mathrm{Ca}+{ }^{48} \mathrm{Ca}$, i.e., about $17 \%$ and $8 \%$, respectively, instead of $30 \%$ and $20 \%$. However, the symmetry energy effect on the neutron-neutron correlation function, which is not affected by Coulomb potential, is not much influenced by the size of reaction system, i.e., it is about $20 \%$ and $15 \%$ for the light and heavy reaction systems, respectively. As in collisions of ${ }^{52} \mathrm{Ca}+{ }^{48} \mathrm{Ca}$, effects due to different isoscalar potentials and $N N$ cross sections on nucleonnucleon correlation functions are found to be small.

\section{SUMMARY AND OUTLOOK}

In conclusion, using an isospin-dependent transport model we explore systematically the isospin effects on nucleonnucleon correlation functions in heavy-ion collisions induced by neutron-rich nuclei at intermediate energies. It is found that the nuclear symmetry energy $E_{\text {sym }}(\rho)$ influences significantly both the emission times and the relative emission sequence of neutrons and protons. A stiffer $E_{\text {sym }}(\rho)$ leads to a faster and almost simultaneous emission of neutrons and protons. Consequently, two-nucleon correlation functions, especially for neutron-proton pairs with high total momentum but low relative momentum, are stronger for the stiff symmetry energy than the soft one. The correlation function of neutronproton pairs gated on higher total momentum but with 
smaller relative momentum is, on the other hand, insensitive to the incompressibility of symmetric nuclear matter EOS and the in-medium $N N$ cross sections. This novel property can be used as a possible tool to extract useful information about the density dependence of nuclear symmetry energy. These results do not change much if only nucleons emitted in certain directions are considered.

We have also studied the dependence of neutron-proton correlation function on the impact parameter and incident energy of heavy-ion collisions as well as masses of the reaction system. We find that the symmetry energy effect becomes weaker with increasing impact parameter and incident energy. Also, the strength of nucleon-nucleon correlation function is reduced in collisions of heavier reaction systems as a result of larger nucleon emission source. For protonproton and neutron-proton correlation functions, the symmetry energy effect is further suppressed by the stronger Coulomb potential in heavier reaction systems. Our results thus suggest that the correlation function of neutron-proton pairs gated on higher total momentum but with smaller relative momentum from central or semicentral collisions induced by lighter neutron-rich nuclei is a sensitive probe to the density dependence of nuclear symmetry energy. We have further studied the dependence of symmetry energy effect on the time at which IBUU simulations are terminated. We find that the symmetry energy effect on nucleon-nucleon correlation functions begins to appear when the maximum nuclear density is already below the normal nuclear matter density. The effect addressed in the present study via nucleon-nucleon correlation functions is thus largely limited to the properties of nuclear symmetry energy at subnormal nuclear densities, similar to that from studying neutron skins of radioactive nuclei.

In the present work, we have not included momentum dependence in either the isoscalar mean-field potential or the symmetry potential. The former may affect the properties of nuclear emission source as it was shown that a momentumdependent mean-field potential reduces nuclear stopping or increases nuclear transparency [76]. With momentum dependence included in the nuclear symmetry potential, differences between the neutron and proton potentials are affected not only by the density dependence of nuclear symmetry energy but also by the magnitude of proton and neutron momenta [77]. It will be of interest to study how the results obtained in the present study are modified by the momentum dependence of nuclear mean field. We plan to carry out such a study in the future.

\section{ACKNOWLEDGMENTS}

This paper was based on the work supported in part by the U.S. National Science Foundation under Grant Nos. PHY0088934 and PHY-0098805 as well as the Welch Foundation under Grant No. A-1358. Also, the work of L.W.C. was supported by the National Natural Science Foundation of China under Grant No. 10105008, and that of V.G. was supported by the National Institute of Nuclear Physics (INFN) in Italy.
[1] Radioactive Nuclear Beams, edited by I. Tanihata [Nucl. Phys. A693, 1 (2001)].

[2] Isospin Physics in Heavy-Ion Collisions at Intermediate Energies, edited by Bao-An Li and W. Udo Schröder (Nova Science, Inc., New York, 2001).

[3] B.A. Li, C.M. Ko, and W. Bauer, Int. J. Mod. Phys. E 7, 147 (1998).

[4] M. Di Toro, V. Baran, M. Colonna, G. Fabbri, A.B. Larionov, S. Maccarone, and S. Scalone, Prog. Part. Nucl. Phys. 42, 125 (1999).

[5] K. Oyamatsu, I. Tanihata, Y. Sugahara, K. Sumiyoshi, and H. Toki, Nucl. Phys. A634, 3 (1998).

[6] B.A. Brown, Phys. Rev. Lett. 85, 5296 (2000).

[7] C.J. Horowitz and J. Piekarewicz, Phys. Rev. Lett. 86, 5647 (2001); Phys. Rev. C 63, 025501 (2001).

[8] R.J. Furnstahl, Nucl. Phys. A706, 85 (2002).

[9] H.A. Bethe, Rev. Mod. Phys. 62, 801 (1990).

[10] J.M. Lattimer and M. Prakash, Astrophys. J. 550, 426 (2001).

[11] I. Bombaci, in Isospin Physics in Heavy-Ion Collisions at Intermediate Energies, edited by Bao-An Li and W. Udo Schröder (Nova Science, New York, 2001), p. 35.

[12] L. Engvik et al., Phys. Rev. Lett. 73, 2650 (1994).

[13] M. Prakash, T.L. Ainsworth, and J. Lattimer, Phys. Rev. Lett. 61, 2518 (1988).

[14] M. Di Toro et al., nucl-th/0210052.

[15] M. Kutschera, Phys. Lett. B 340, 1 (1994).
[16] S. Kubis and M. Kutschera, astro-ph/0207490.

[17] B.A. Li, C.M. Ko, and Z.Z. Ren, Phys. Rev. Lett. 78, 1644 (1997).

[18] B.A. Li and C.M. Ko, Nucl. Phys. A618, 498 (1997).

[19] V. Baran, M. Colonna, M. Di Toro, and A.B. Larionov, Nucl. Phys. A632, 287 (1998).

[20] H.S. Xu et al., Phys. Rev. Lett. 85, 716 (2000).

[21] W.P. Tan et al., Phys. Rev. C 64, 051901(R) (2001).

[22] V. Baran, M. Colonna, M. Di Toro, V. Greco, M. ZielinskaPfabé, and H.H. Wolter, Nucl. Phys. A703, 603 (2002).

[23] M.B. Tsang et al., Phys. Rev. Lett. 86, 5023 (2001).

[24] B.A. Li, A.T. Sustich, and B. Zhang, Phys. Rev. C 64, 054604 (2001).

[25] B.A. Li, Phys. Rev. Lett. 85, 4221 (2000).

[26] B.A. Li, Phys. Rev. Lett. 88, 192701 (2002); Nucl. Phys. A708, 365 (2002); Phys. Rev. C 67, 017601 (2003).

[27] D.H. Boal, C.K. Gelbke, and B.K. Jennings, Rev. Mod. Phys. 62, 553 (1990).

[28] W. Bauer, C.K. Gelbke, and S. Pratt, Annu. Rev. Nucl. Part. Sci. 42, 77 (1992).

[29] D. Ardouin, Int. J. Mod. Phys. E 6, 391 (1997).

[30] U.A. Wiedemann and U. Heinz, Phys. Rep. 319, 145 (1999).

[31] C.J. Gelderloos et al., Phys. Rev. Lett. 75, 3082 (1995).

[32] R. Lednicky, V.L. Lyuboshitz, B. Erazmus, and D. Nouais, Phys. Lett. B 373, 30 (1996).

[33] S. Voloshin, R. Lednicky, S. Panitkin, and N. Xu, Phys. Rev. Lett. 79, 4766 (1997). 
[34] D. Ardouin et al., Phys. Lett. B 446, 191 (1999).

[35] F. Wang, and S. Pratt, Phys. Rev. Lett. 83, 3138 (1999).

[36] D. Gourio et al., Eur. Phys. J. A 7, 245 (2000).

[37] R. Kotte et al., The FOPI Collaboration, Eur. Phys. J. A 6, 185 (1999).

[38] L.W. Chen, V. Greco, C.M. Ko, and B.A. Li, Phys. Rev. Lett. 90, 162701 (2003); nucl-th/0211002.

[39] R.B. Wiringa, V. Fiks, and A. Fabrocini, Phys. Rev. C 38, 1010 (1988).

[40] J.M. Lattimer, C.J. Pethick, M. Prakash, and P. Haensel, Phys. Rev. Lett. 66, 2701 (1991).

[41] P.J. Siemens, Nucl. Phys. A141, 225 (1970).

[42] G. Baym, H.A. Bethe, and C.J. Pethick, Nucl. Phys. A175, 225 (1971).

[43] M. Prakash, T.L. Ainsworth, and J.M. Lattimer, Phys. Rev. Lett. 61, 2518 (1988).

[44] V. Thorsson, M. Prakash, and J.M. Lattimer, Nucl. Phys. A572, 69 (1994).

[45] P.E. Haustein, At. Data Nucl. Data Tables 39, 185 (1988).

[46] I. Bombaci and U. Lombardo, Phys. Rev. C 44, 1892 (1991).

[47] S. Shlomo and D.H. Youngblood, Phys. Rev. C 47, 529 (1993).

[48] H. Heiselberg and M. Hjorth-Jensen, Phys. Rep. 328, 237 (2000).

[49] L.W. Chen, F.S. Zhang, Z.H. Lu, W.F. Li, Z.Y. Zhu, and H.R. Ma, J. Phys. G 27, 1799 (2001).

[50] G.F. Bertsch and S. Das Gupta, Phys. Rep. 160, 189 (1988).

[51] B.A. Li, Z.Z. Ren, C.M. Ko, and S.J. Yennello, Phys. Rev. Lett. 76, 4492 (1996); B.A. Li and A.T. Sustich, ibid. 82, 5004 (1999).

[52] B.D. Serot and J.D. Walecka, Adv. Nucl. Phys. 16, 1 (1986).

[53] Z.Y. Zhu, W.Q. Shen, Y.H. Cai, and Y.G. Ma, Phys. Lett. B 328, 1 (1994).
[54] Z. Ren et al., Phys. Rev. C 52, R20 (1995).

[55] Q. Pan and P. Danielewicz, Phys. Rev. Lett. 70, 2062 (1993).

[56] J. Zhang, S. Das Gupta, and C. Gale, Phys. Rev. C 50, 1617 (1994).

[57] G.Q. Li and R. Machleidt, Phys. Rev. C 48, 1702 (1993); 49, 566 (1994).

[58] P. Danielewicz, Nucl. Phys. A685, 368c (2001).

[59] D.O. Handzy et al., Phys. Rev. Lett. 75, 2916 (1995).

[60] B.A. Li and S.J. Yennello, Phys. Rev. C 52, R1746 (1995).

[61] G. Bertsch, P. Danielewicz, and H. Schulz, Europhys. Lett. 21, 817 (1993).

[62] B. Erazmus et al., Nucl. Phys. A583, 395 (1995).

[63] L.G. Moretto, K. Tso, N. Colonna, and G.J. Wozniak, Phys. Rev. Lett. 69, 1884 (1992).

[64] W. Bauer, G.F. Bertsch, and H. Schulz, Phys. Rev. Lett. 69, 1888 (1992).

[65] D.O. Handzy et al., Phys. Rev. C 51, 2237 (1995).

[66] W.G. Gong et al., Phys. Rev. Lett. 65, 2114 (1990).

[67] W.G. Gong, W. Bauer, C.K. Gelbke, and S. Pratt, Phys. Rev. C 43, 781 (1991).

[68] W.G. Gong et al., Phys. Rev. C 47, R429 (1993).

[69] G.J. Kunde et al., Phys. Rev. Lett. 70, 2545 (1993).

[70] R. Ghetti et al., Nucl. Phys. A674, 277 (2000).

[71] R. Ghetti et al., Phys. Rev. Lett. 87, 102701 (2001).

[72] S.E. Koonin, Phys. Lett. 70B, 43 (1977).

[73] S. Pratt, Phys. Rev. Lett. 53, 1219 (1984); Phys. Rev. D 33, 72 (1986).

[74] S. Pratt and M.B. Tsang, Phys. Rev. C 36, 2390 (1987).

[75] S. Pratt, Nucl. Phys. A566, 103c (1994).

[76] V. Greco, A. Guarnera, M. Colonna, and M. Di Toro, Phys. Rev. C 59, 810 (1999).

[77] C.B. Das, S. Das Gupta, C. Gale, and B.A. Li, Phys. Rev. C 67, 034611 (2003). 